\title{
CLIMATIC DATA FOR WILLIAMS LAKE, HUBBARD COUNTY, MINNESOTA, 1982
}

By

D.O. Rosenberry, A.M. Sturrock, J. L. Scarborough and T.C. Winter

DEPARTMENT OF THE INTERIOR

U.S. GEOLOGICAL SURVEY

Open-File Report 88-89

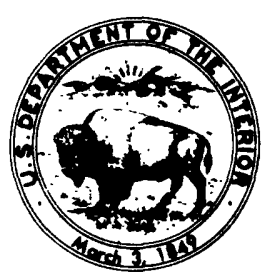




\author{
DEPARTMENT OF THE INTERIOR \\ DONALD PAUL HODEL, Secretary \\ U.S. GEOLOGICAL SURVEY \\ Dallas L. Peck, Director
}

\begin{tabular}{ll}
\hline For additional information & $\begin{array}{l}\text { Copies of this report can } \\
\text { be purchased from: }\end{array}$ \\
Thomas C. Winter & U.S. Geological Survey \\
U.S. Geological Survey & Books and Open-File Reports \\
Box 25046, MS 413 & Section \\
Denver Federal Center & $\begin{array}{l}\text { Federal Center, B1dg. } 810 \\
\text { Denver, CO } 80225\end{array}$ \\
& Box 25425 \\
& Denver, Co 80225
\end{tabular}




\section{CONTENTS}

Page

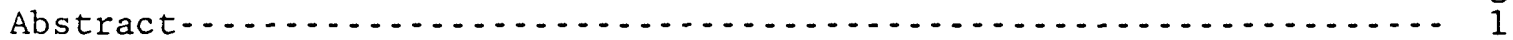

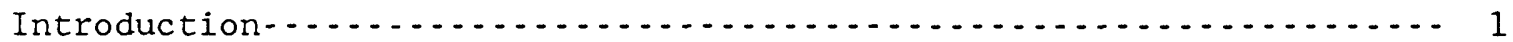

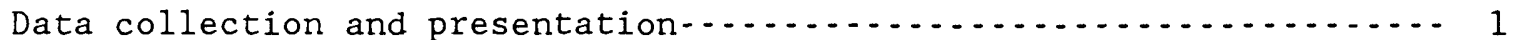

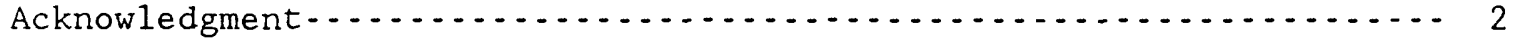

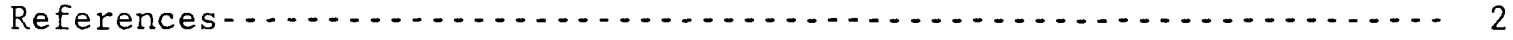

TABLES

Table 1. Summary of 1982 energy-budget data................... 3

2. Summary of 1982 temperature data at the raft station..... 14

3. Summary of 1982 wind-speed data at the raft station...... 19

4. Summary of 1982 radiation data at the land station....... 29

5. Summary of 1982 temperature data at the land station-.... 34

6. Summary of 1982 wind-speed data at the land station-.... 39 
METRIC CONVERSION FACTORS

$\begin{array}{lll}\text { Multiply } & \text { By } & \text { To obtain } \\ \text { meter } & 3.281 & \text { feet } \\ \text { kilometer } & 0.621 & \text { mile } \\ \text { centimeter } & 0.394 & \text { inch } \\ \text { millibar } & 0.0145 & \text { pound per square inch } \\ \text { millibar } & 1.0197 & \text { grams per square centimeter } \\ \text { mile per hour } & 1.609 & \text { kilometer per hour } \\ \text { calories per square centimeter } & 1.433 \times 10-3 & \text { watts per square meter } \\ \quad \text { per minute } & & \text { watts per square meter } \\ \begin{array}{l}\text { calories per square centimeter } \\ \quad 278.96\end{array} & \text { watts per square foot } \\ \begin{array}{l}\text { calories per square centimeter } \\ \quad \text { per day }\end{array} & 25.913 & \\ \begin{array}{l}\text { To convert degrees Celsius }\left({ }^{\circ} \mathrm{C}\right) \\ \text { formula: }\end{array} & \end{array}$


CLIMATIC DATA FOR WILLIAMS LAKE, HUBBARD COUNTY, MINNESOTA

1982

by D.O. Rosenberry, A.M. Sturrock,

J.L. Scarborough, and T.C. Winter

\begin{abstract}
Research on the hydrology of Williams Lake, north-central Minnesota includes study of evaporation. Presented here are those climatic data needed for energy-budget and mass-transfer studies, including: watersurface temperature, dry-bulb and wet-bulb air temperatures, wind speed, precipitation, and solar and atmospheric radiation. Some calculated values necessary for these studies are also presented, such as vapor pressure and Bowen-ratio values. Data are collected at raft and land stations.
\end{abstract}

\title{
INTRODUCTION
}

Climatic data are being collected at Williams Lake, Hubbard County, Minnesota, as part of a continuing study by the U.S. Geological Survey of the hydrology of the lake. Williams Lake is one of several lakes in different parts of the United States that have been selected for intensive study of hydrological and related chemical and biological processes. The rationale for selection of Williams Lake is given by Siegel and Winter (1980). Climatic data collected during 1983 through 1986 are presented in Rosenberry and others (1988) and in Sturrock and others (1984, 1986a, 1986b).

\section{DATA COLLECTION AND PRESENTATION}

Data presented here are being collected principally for studies of evaporation. Therefore, the period of record includes only the time when the lake is ice-free. The period of record for 1982 is from June 13 (Julian day 164) to October 24 (Julian day 297). The interval from day 164 to day 297 is the part of the year for which evaporation will be determined by the energy-budget method. Within each table, the data are grouped into energybudget periods, which are defined by the dates thermal surveys were made of the lake. For example, the first energy-budget period is from Julian day 164 through Julian day 176.

Climatic instruments are located on a raft near the middle of the lake, and at a land station located near the northwest shore of the lake.

Instruments on the raft include anemometers at 1,2 , and 4 meters above the water surface, a thermistor psychrometer with wet-and dry-bulb temperature sensors fixed at 2 meters above the water surface, and a water-temperature sensor located beneath the raft at a depth of about 1 centimeter. Data from the above sensors are recorded by a digtital data logger located on the raft. The data logger scans the sensors every minute and calculates hourly 
and daily averages. In addition, for selected sensors, the daily output includes maximum and minimum values and the time they occur.

The land station consists of long- and short-wave radiometers; an anemometer and thermistor psychrometer, both at 2 meters above the land surface; and a tipping-bucket rain gage. Data from these sensors are recorded by a data logger, identical to that on the raft, which records hourly totals and averages, and daily totals, averages, and selected maximum and minimum values and their associated times of occurence. Additional backup instruments include an analog hygrothermograph and a manually read rain gage.

Calibration checks are made every few days .t both stations using independent laboratory thermometers and wind-up and motorized psychrometers.

Data presented here are daily summaries. For periods that the primary instruments were not operating properly, daily values were obtained by regression using data from backup instruments, provided a satisfactory statistical relation could be established. Data used to establish regressions were selected to bracket the period of missing or inadequate data. Only table 1 , which is considered to be the primary source of data for evaporation studies, includes values obtained by regression.

Although only daily values are reported here, hourly values also were recorded. Hourly values are voluminous and expensive to reproduce, but they are available for all or part of the period of record on request.

\section{ACKNOWLEDGMENT}

We are especially indebted to Kenneth Chase for allowing us to place climatic instruments on his property.

\section{REFERENCES}

Koberg, G.E., 1962, Methods to compute long-wave radiation from the atmosphere and reflected solar radiation from a water surface: U.S. Geological Survey Professional Paper 272-F, p. 107-112.

Rosenberry, D.O., Sturrock, A.M, and Winter, T.C., 1988, Climatic data for Williams Lake, Hubbard County, Minnesota, 1986: U.S. Geological Survey Open-File Report 88-304, 39 p.

Siege1, D.I., and Winter, T.C., 1980, Hydrologic setting of Williams Lake, Hubbard County, Minnesota: U.S. Geological Survey Open-File Report 80 403, $56 \mathrm{p}$.

Sturrock, A.M., Rosenberry, D.O., Englebrecht, L.G., Gothard, W.A., and Winter, T.C., 1984, Climatic data for Williams Lake, Hubbard County, Minnesota, 1983: U.S. Geological Survey Open-File Report 84-247, 41 p.

Sturrock, A.M., Rosenberry, D.O., Scarborough, J.L., and Winter, T.C., 1986a, Climatic data for Williams Lake, Hubbard County, Minnesota, 1984: U.S. Geological Survey Open-File Report 86-64, 63 p.

Sturrock, A.M., Rosenberry, D.0., and Winter, T.C., 1986b, Climatic data for Williams Lake, Hubbard County, Minnesota, 1985: U.S. Geological Survey Open-File Report 86-607, 40 p. 


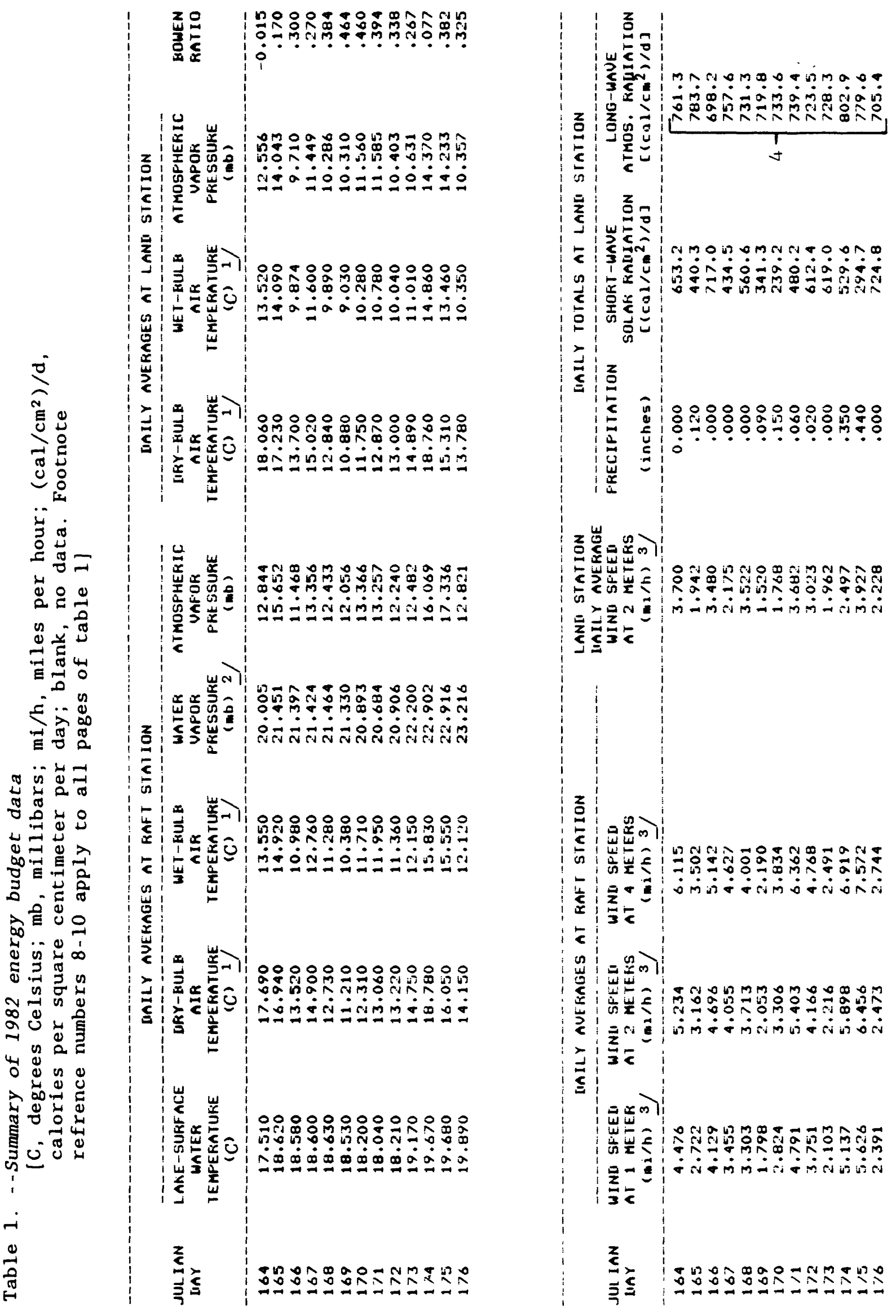




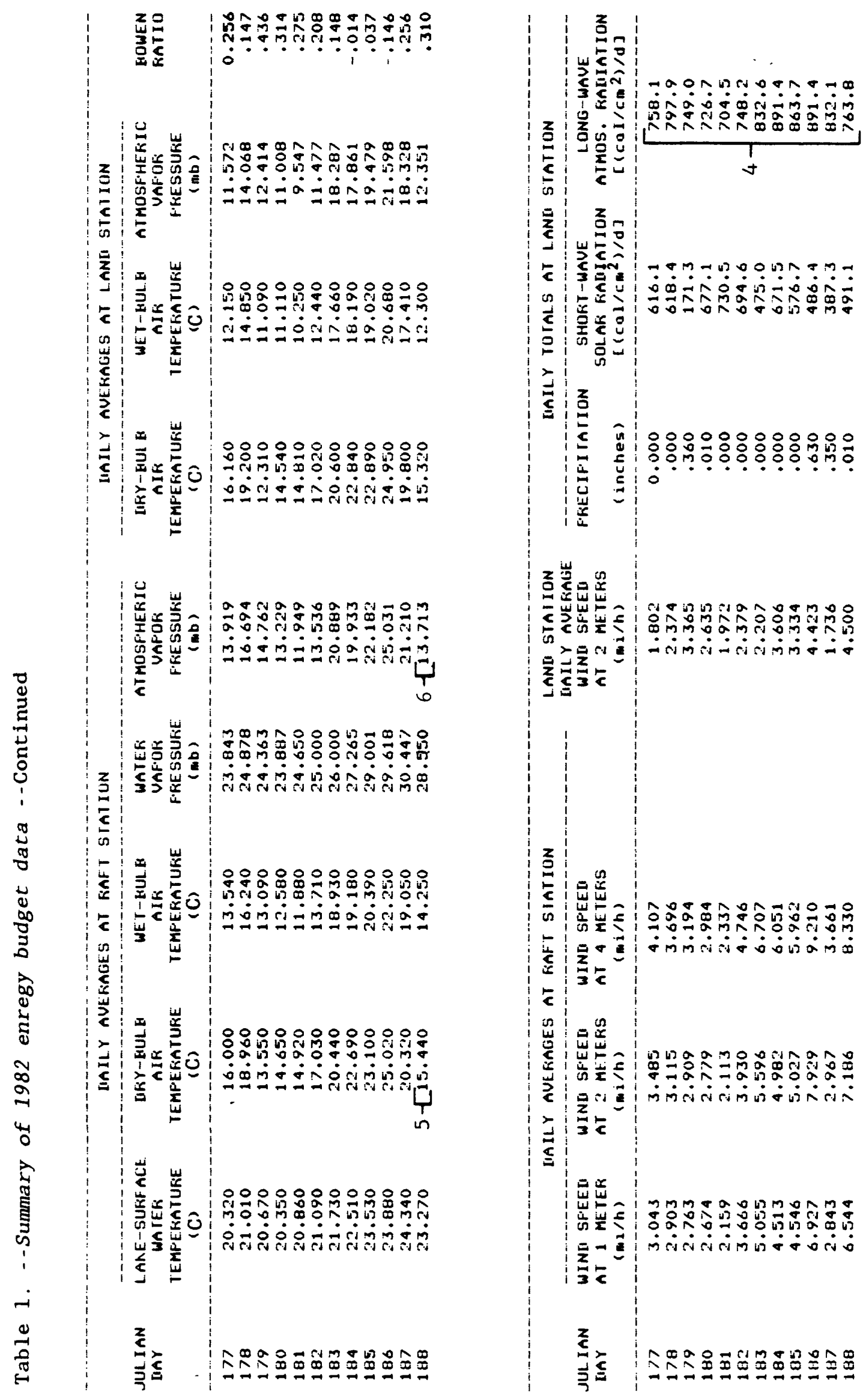



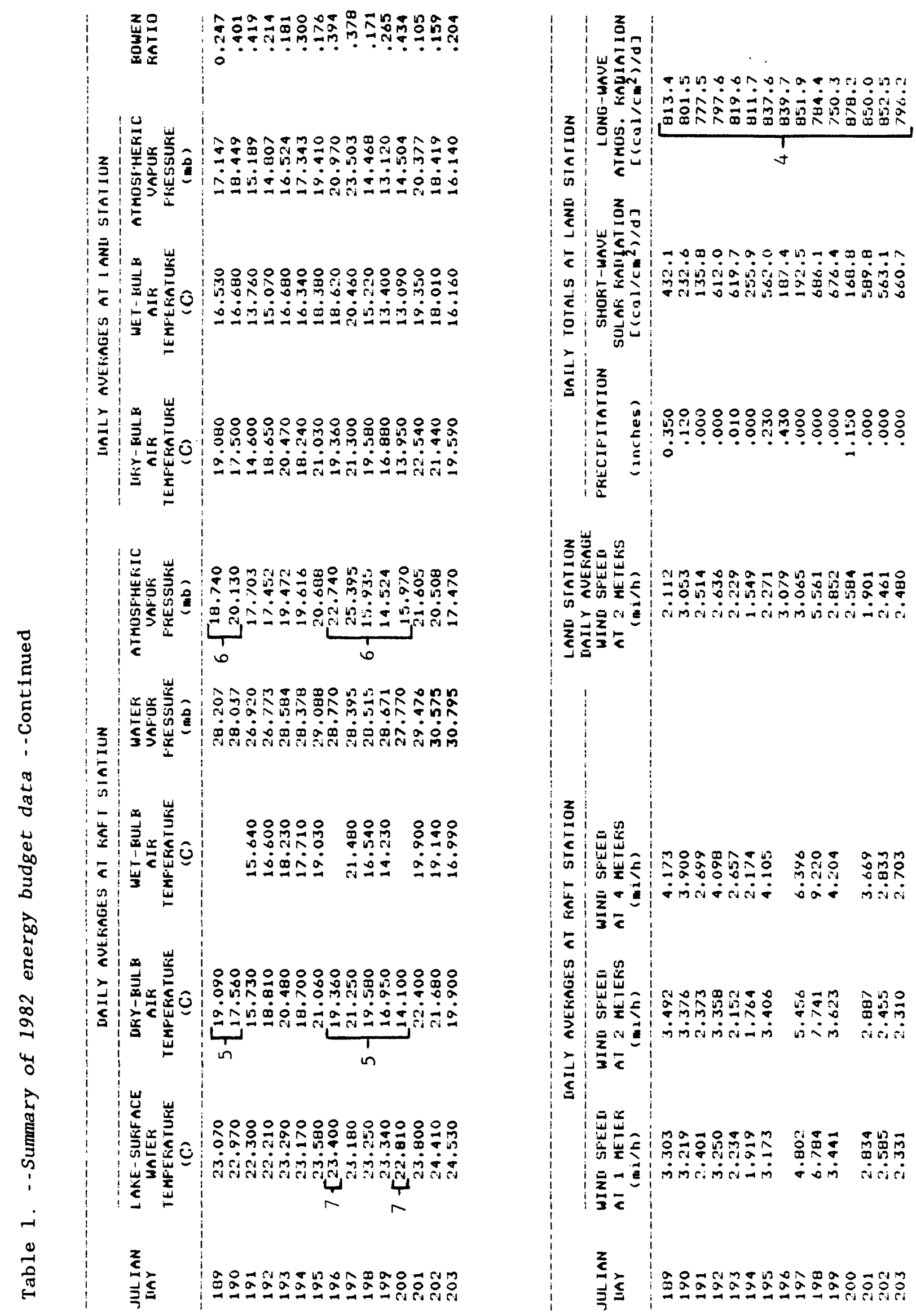

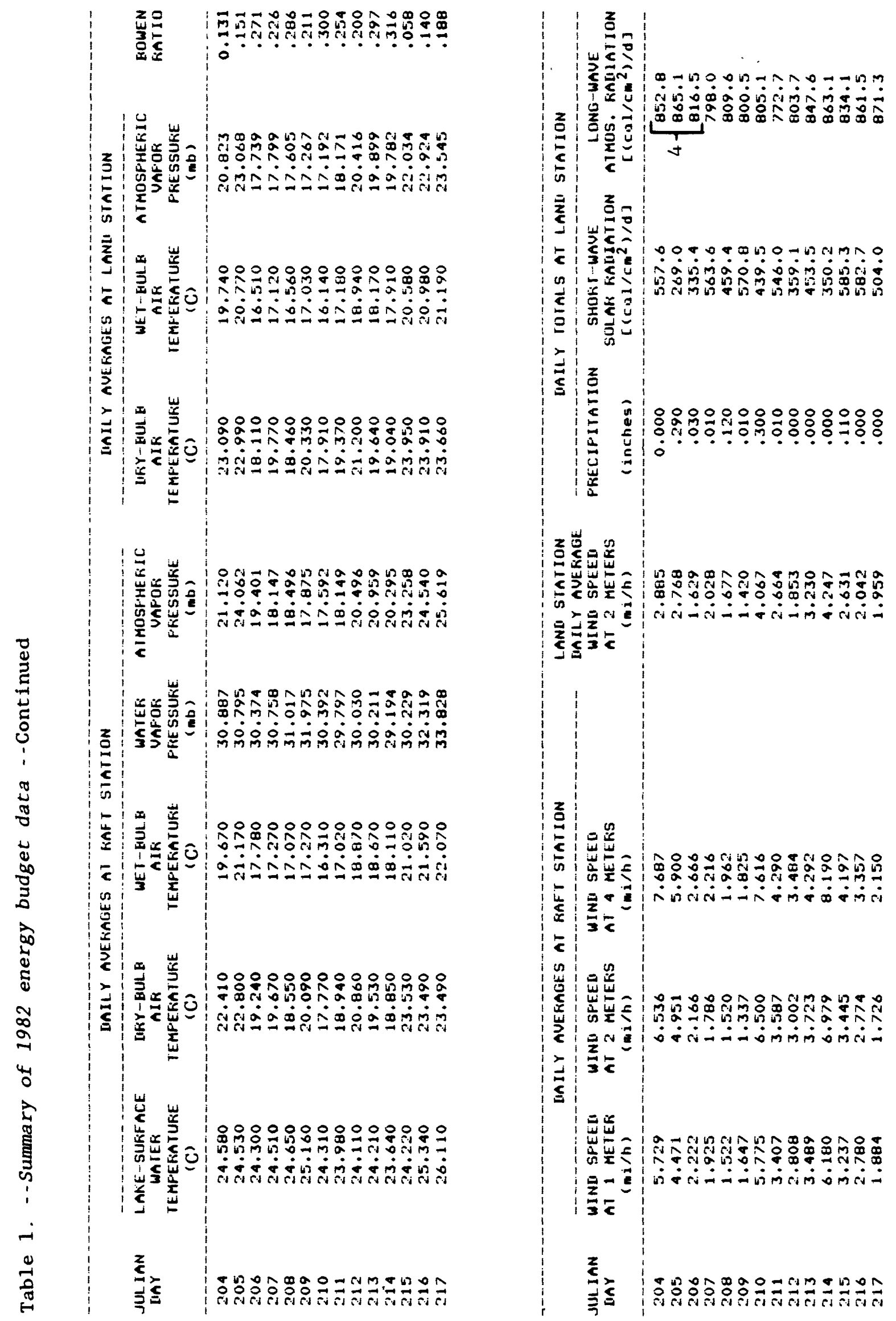


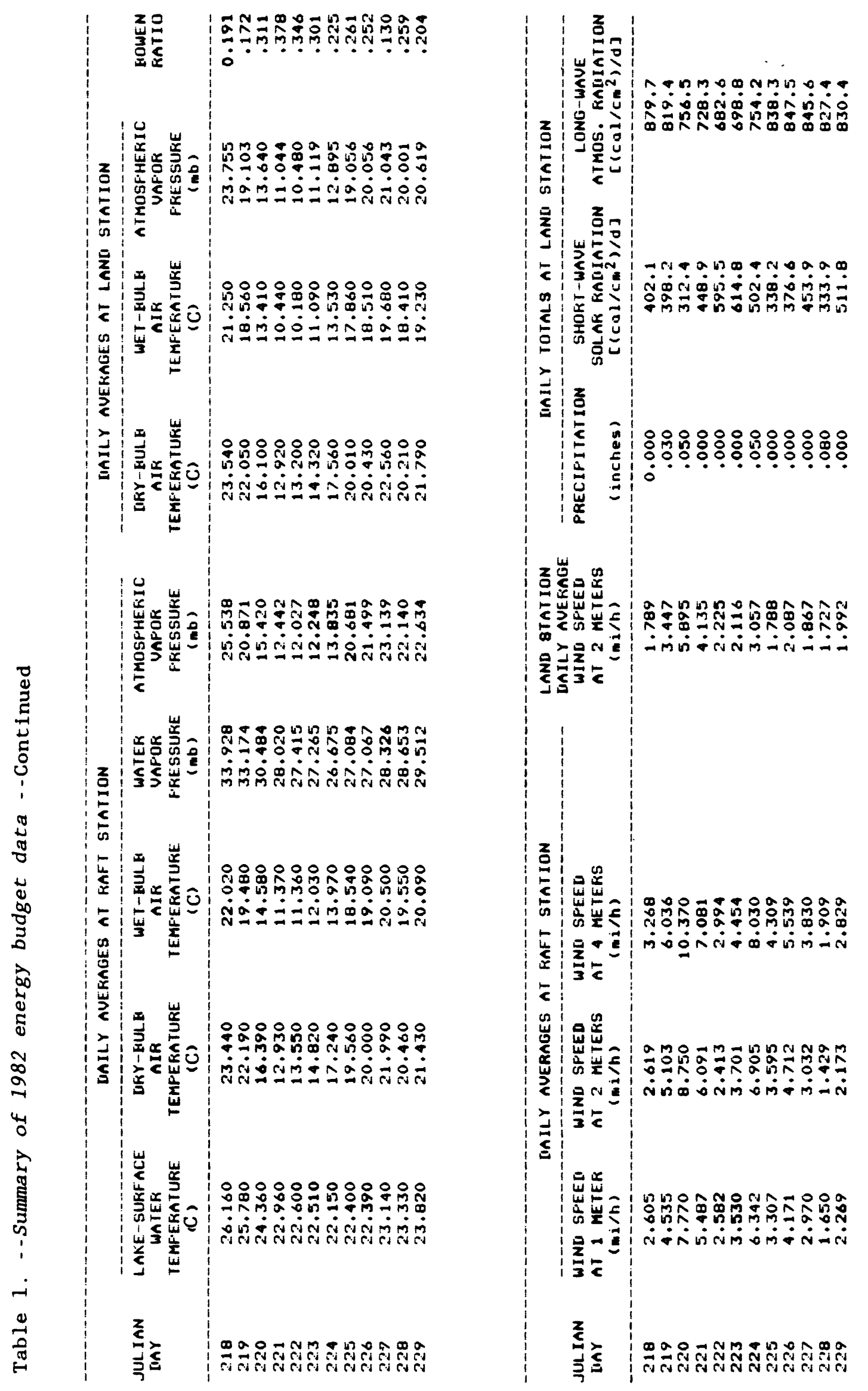




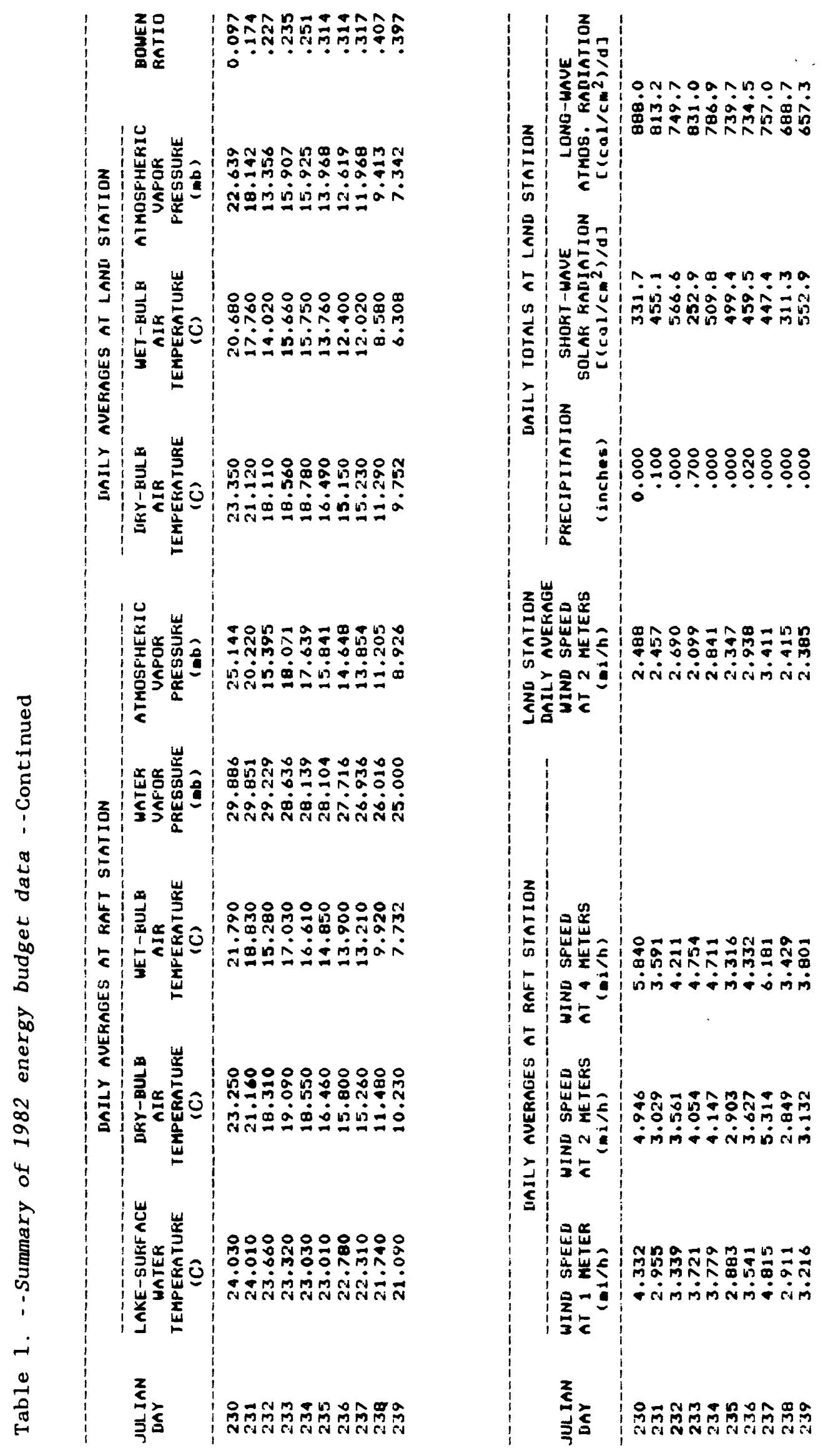



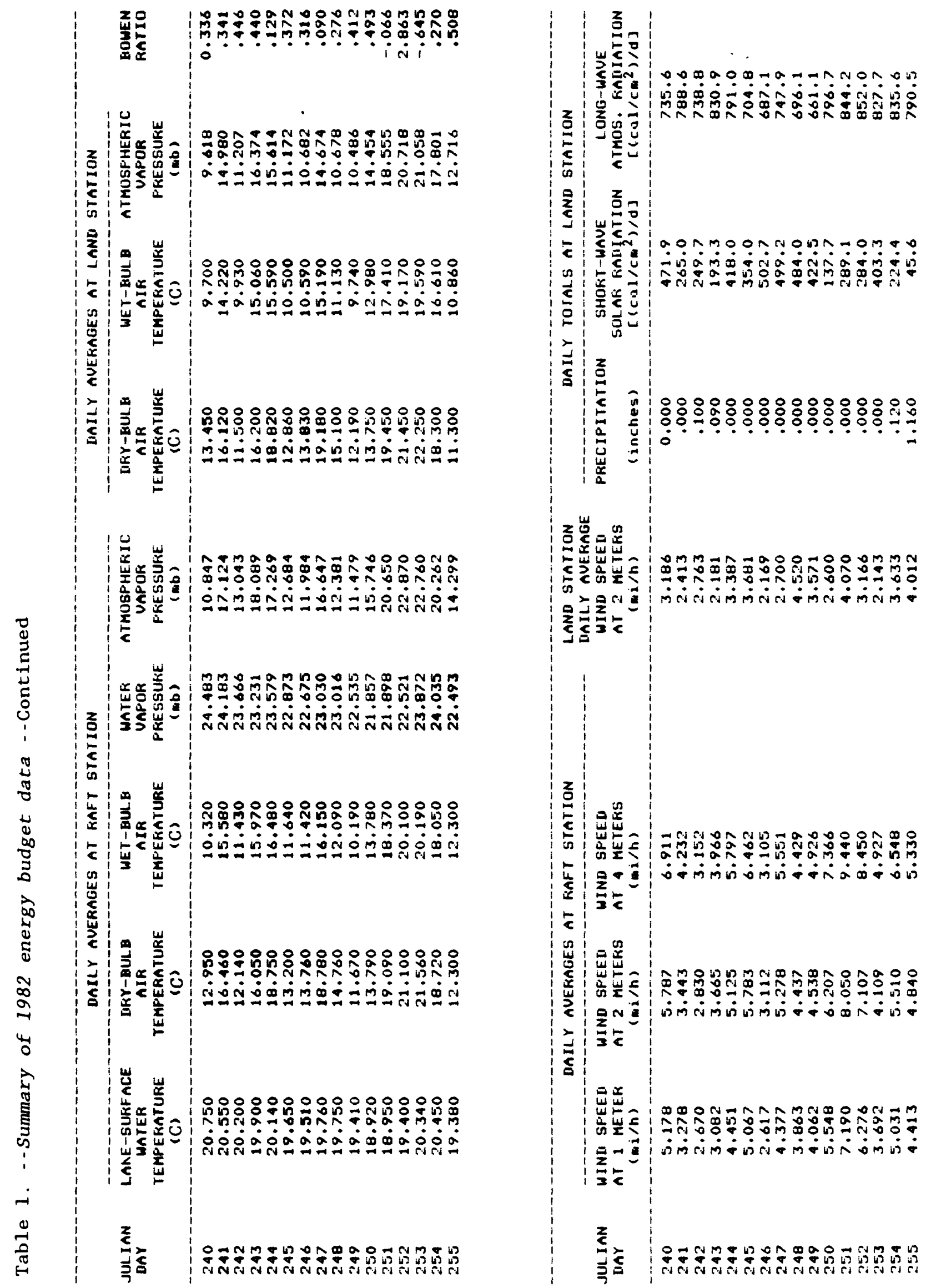

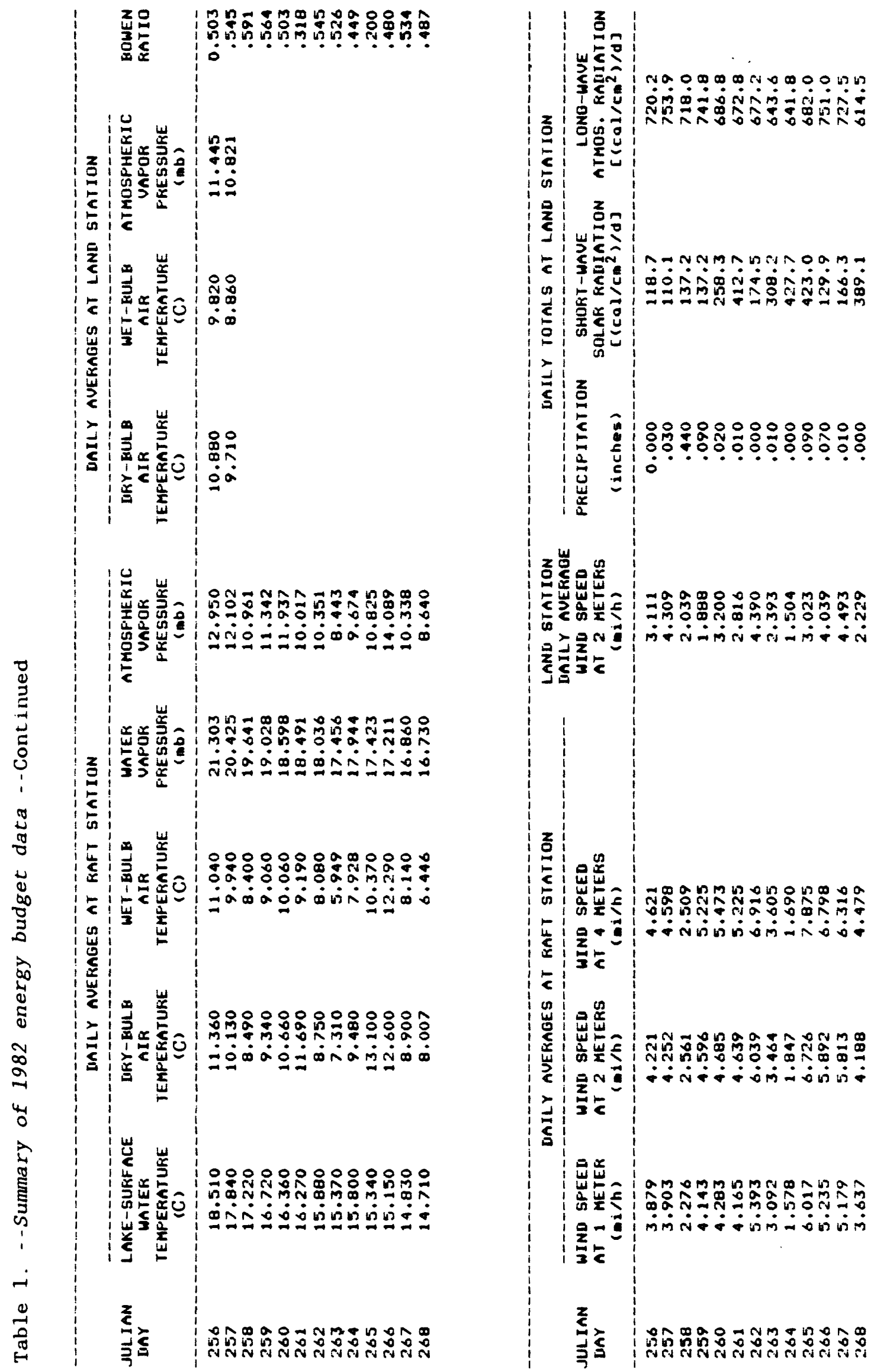

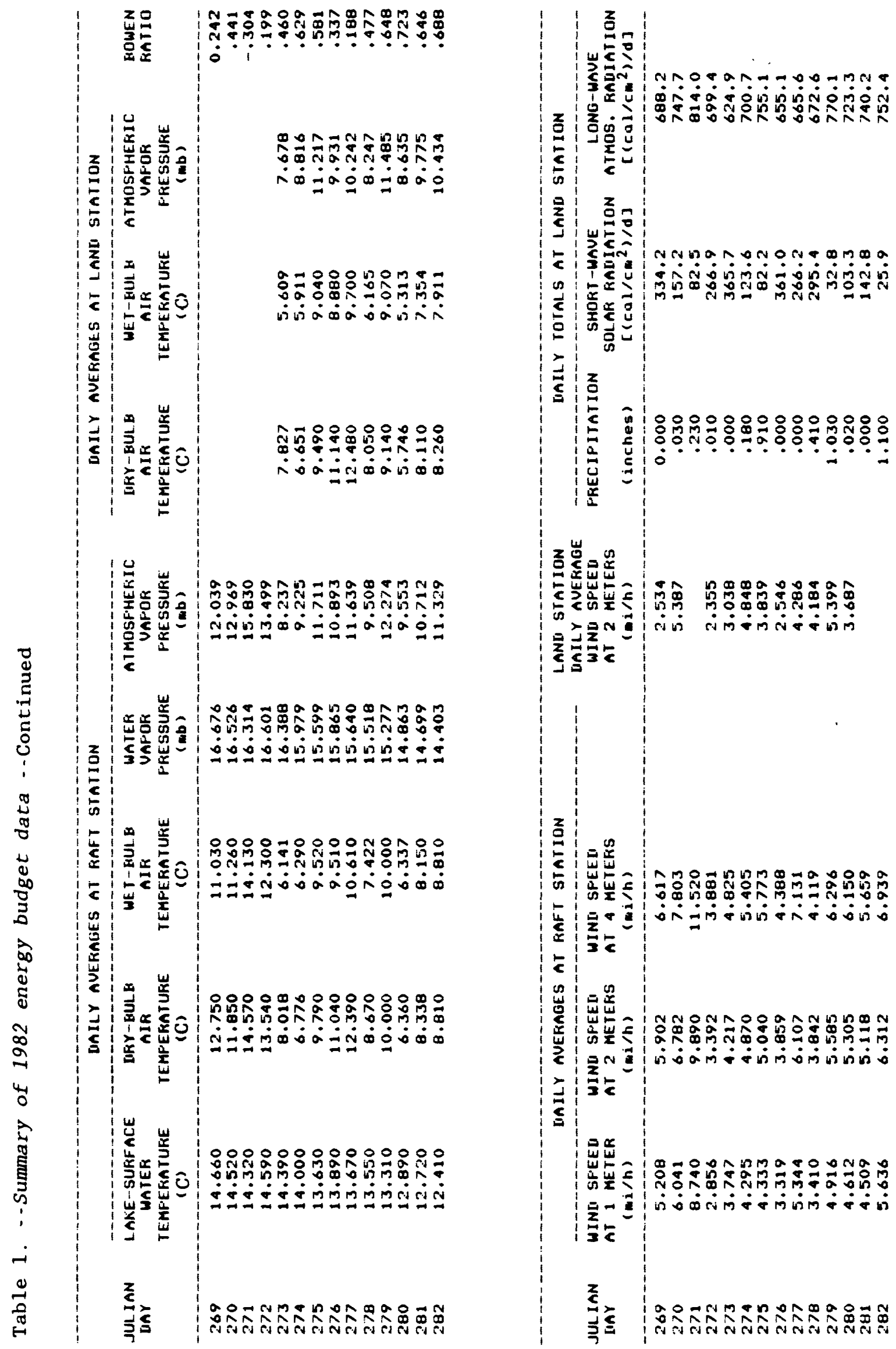

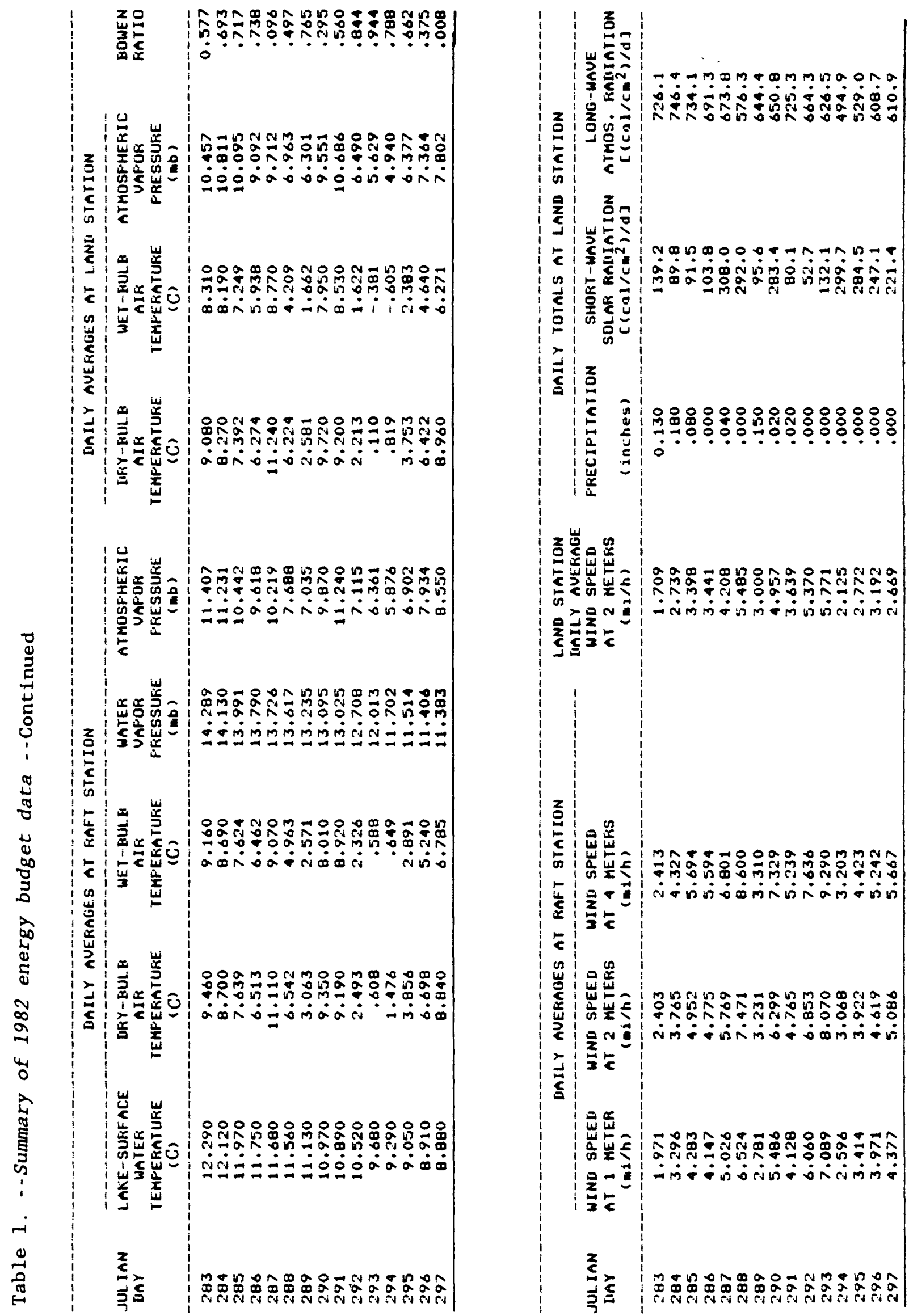
Footnotes to table 1:

${ }^{1}$ Measured at 2 meters above water or 1 and surface.

${ }^{2}$ Water-vapor pressure is calculated using surface water-temperature data and assuming the air is completely saturated at the air-water interface.

${ }^{3}$ Elevation above water or land surface.

4 Calculated by regression equation 1 (see below), which was determined by using Brunt's equation (Koberg, 1964) and the atmospheric long-wave radiation sensor connected to the digital-data logger.

${ }^{5} \mathrm{Calculated}$ by regression equation 2 (see below), which was determined by using air-temperature data from the dry-bulb sensors connected to digital-data loggers at the raft and land stations.

${ }^{6} \mathrm{Calculated}$ by regression equation 3 (see below), which was determined by using calculated vapor pressures at the raft and land stations.

${ }^{7}$ Three hourly values were interpolated from a 21 hour trend.

\begin{tabular}{ccc}
\hline Regression & Data points & $\mathrm{r}^{2}$ value \\
\hline 1. $\mathrm{Y}_{1}=79.070+1.015 \mathrm{X}_{1}$ & 90 & 0.862 \\
2. $\mathrm{Y}_{2}=0.545+0.972 \mathrm{X}_{2}$ & 111 & 0.996 \\
3. $\mathrm{Y}_{3}=0.787+1.047 \mathrm{X}_{3}$ & 111 & 0.971 \\
\hline
\end{tabular}

$Y_{1}=$ atmospheric long-wave radiation measured by the sensor at the land station.

$\mathrm{X}_{1}=$ atmospheric long-wave radiation derived from Brunt's equation.

$\mathrm{Y}_{2}=\mathrm{dry}-\mathrm{bulb}$ air temperature measured by the sensor at the raft station.

$\mathrm{X}_{2}=\mathrm{dry}$-bulb air temperature measured by the sensor at the land station.

$\mathrm{Y}_{3}=$ calculated vapor pressure using data gathered at the raft station.

$\mathrm{X}_{3}=$ calculated vapor pressure using data gathered at the land station. 

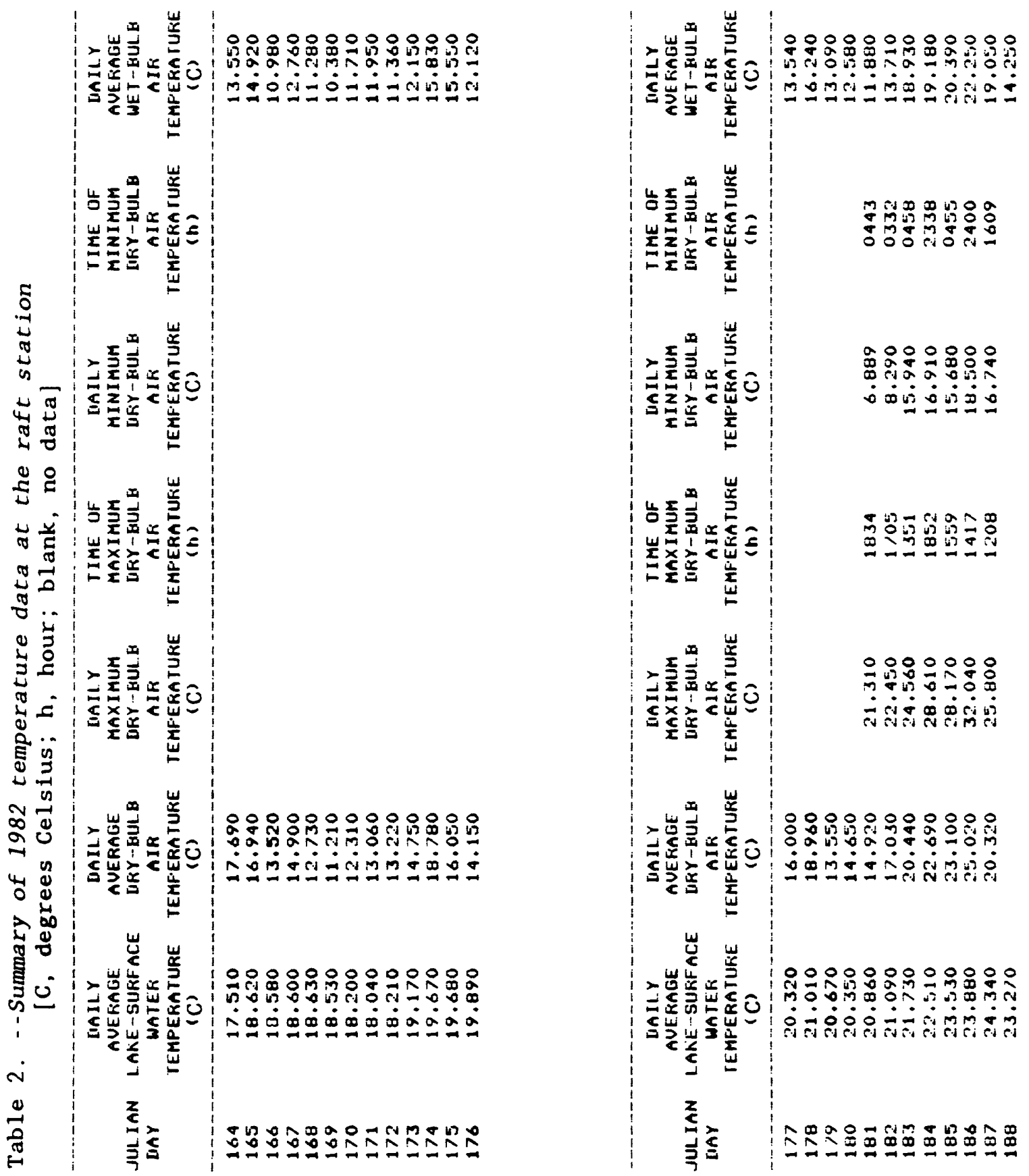

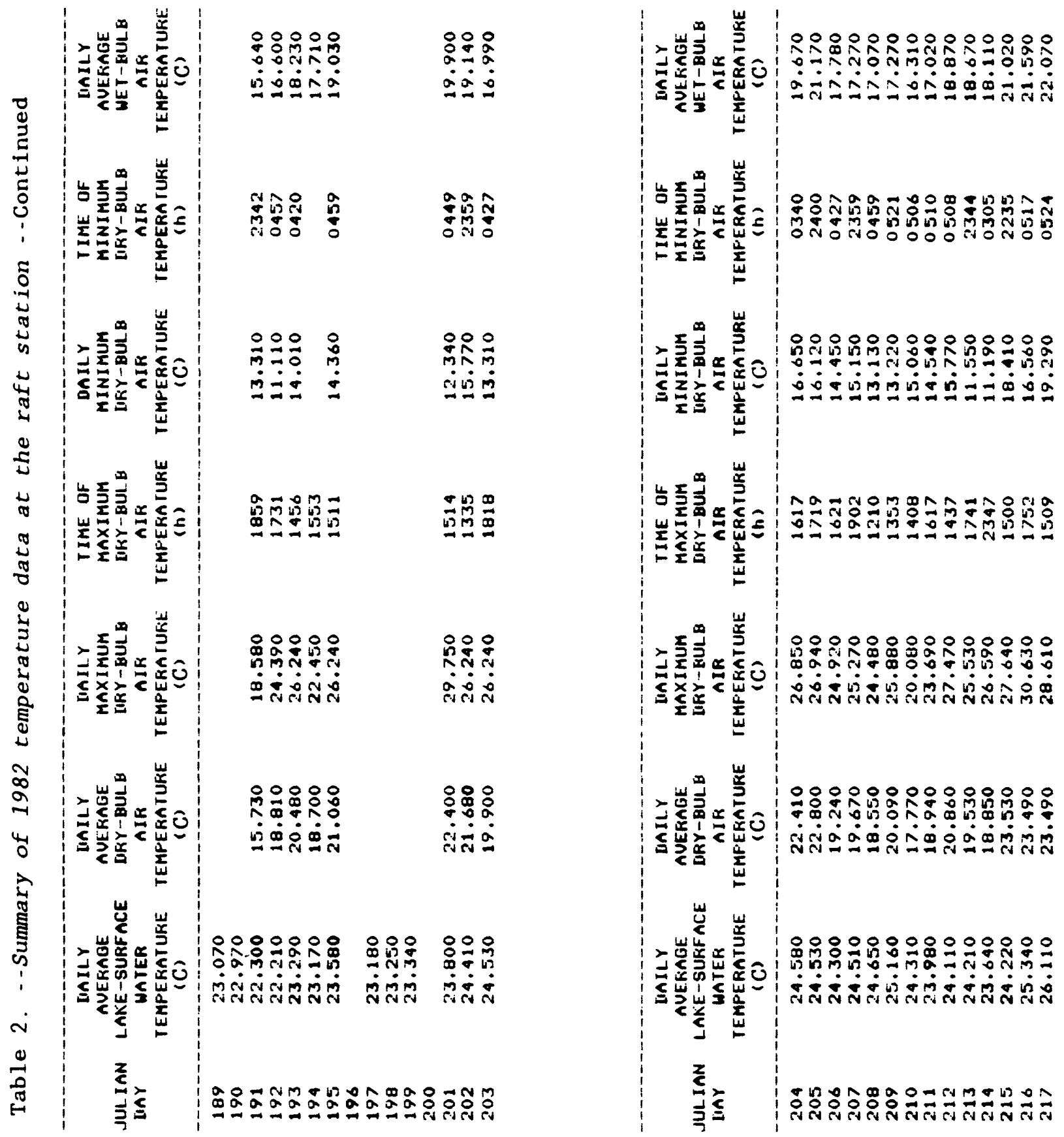

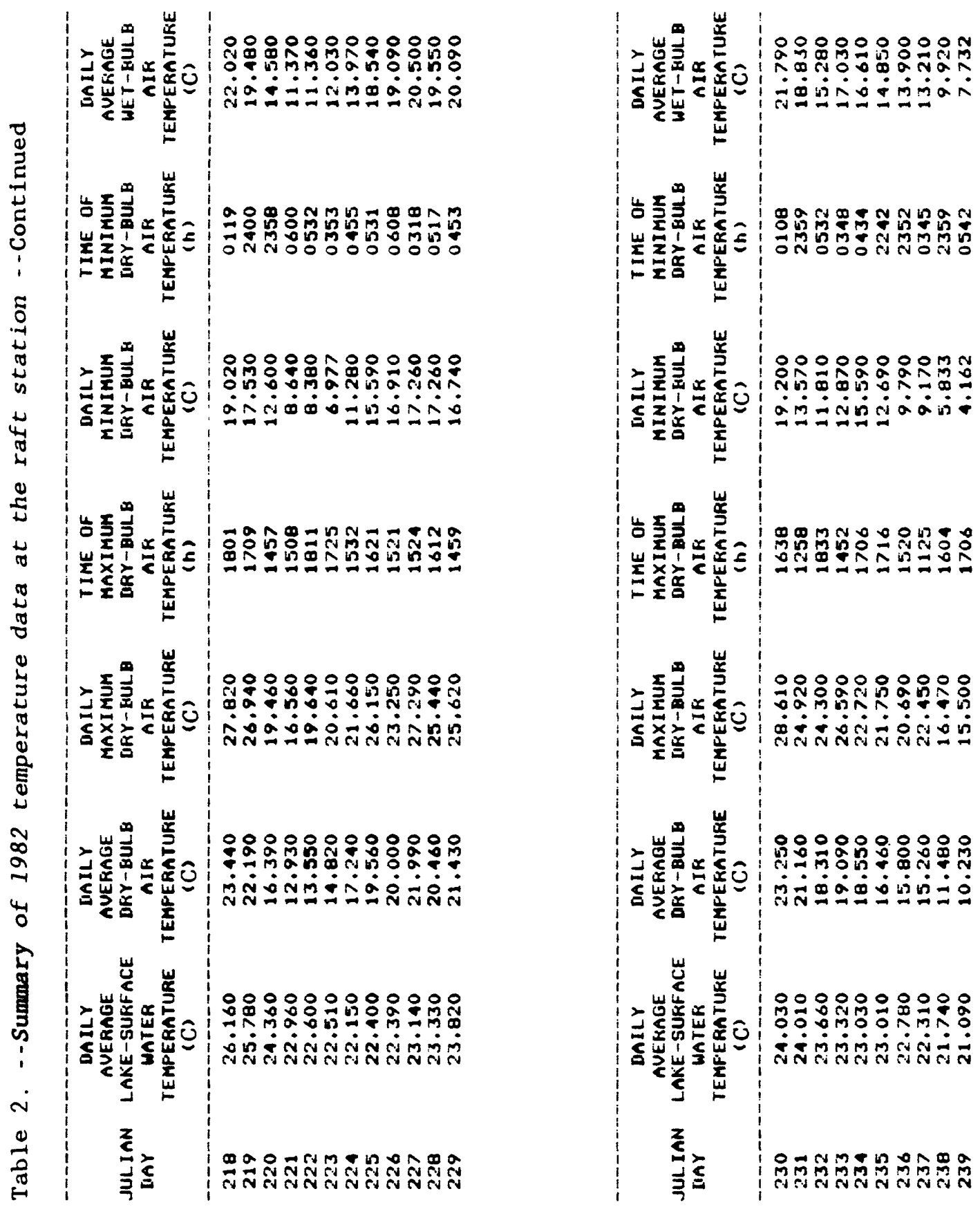

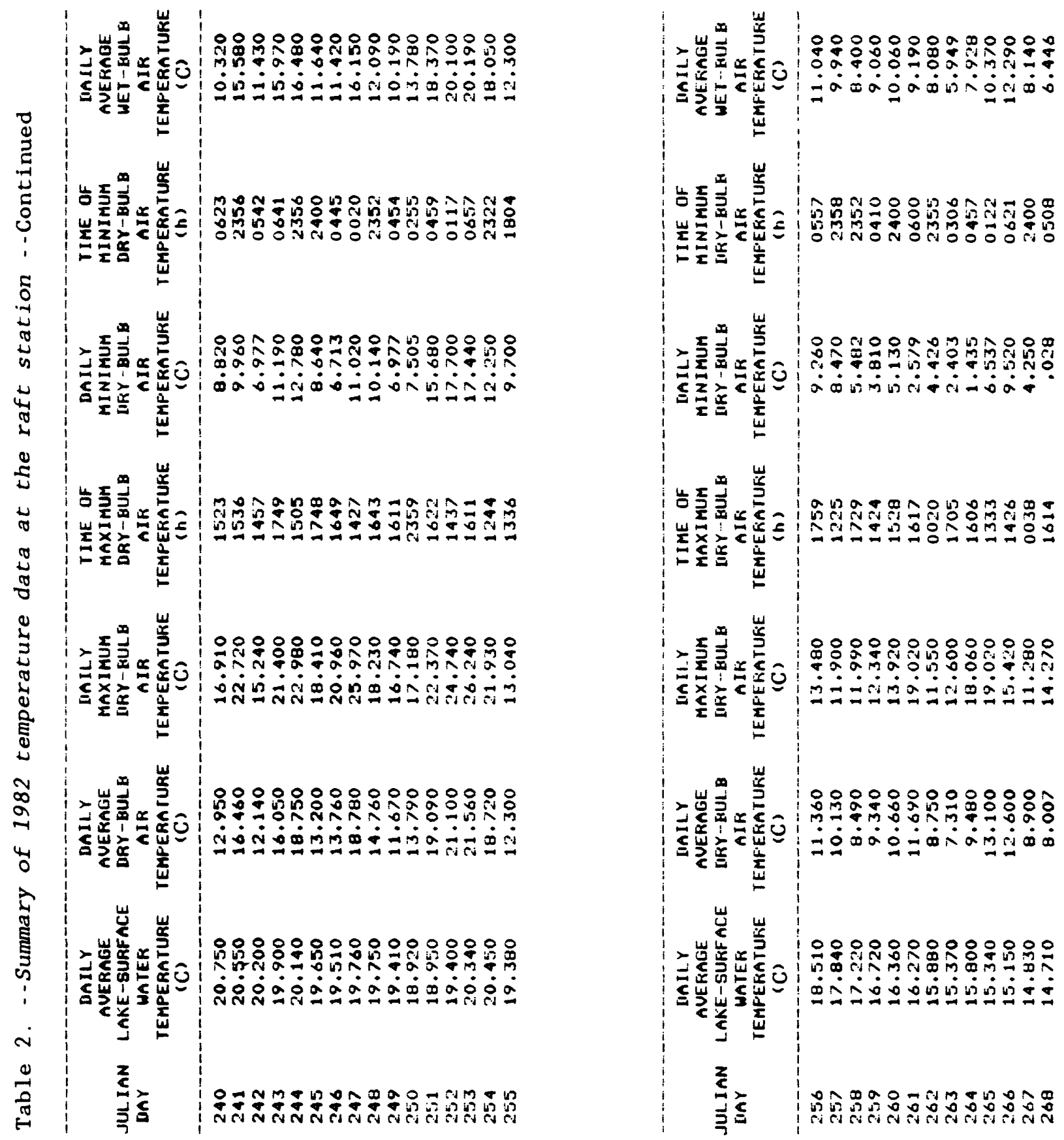

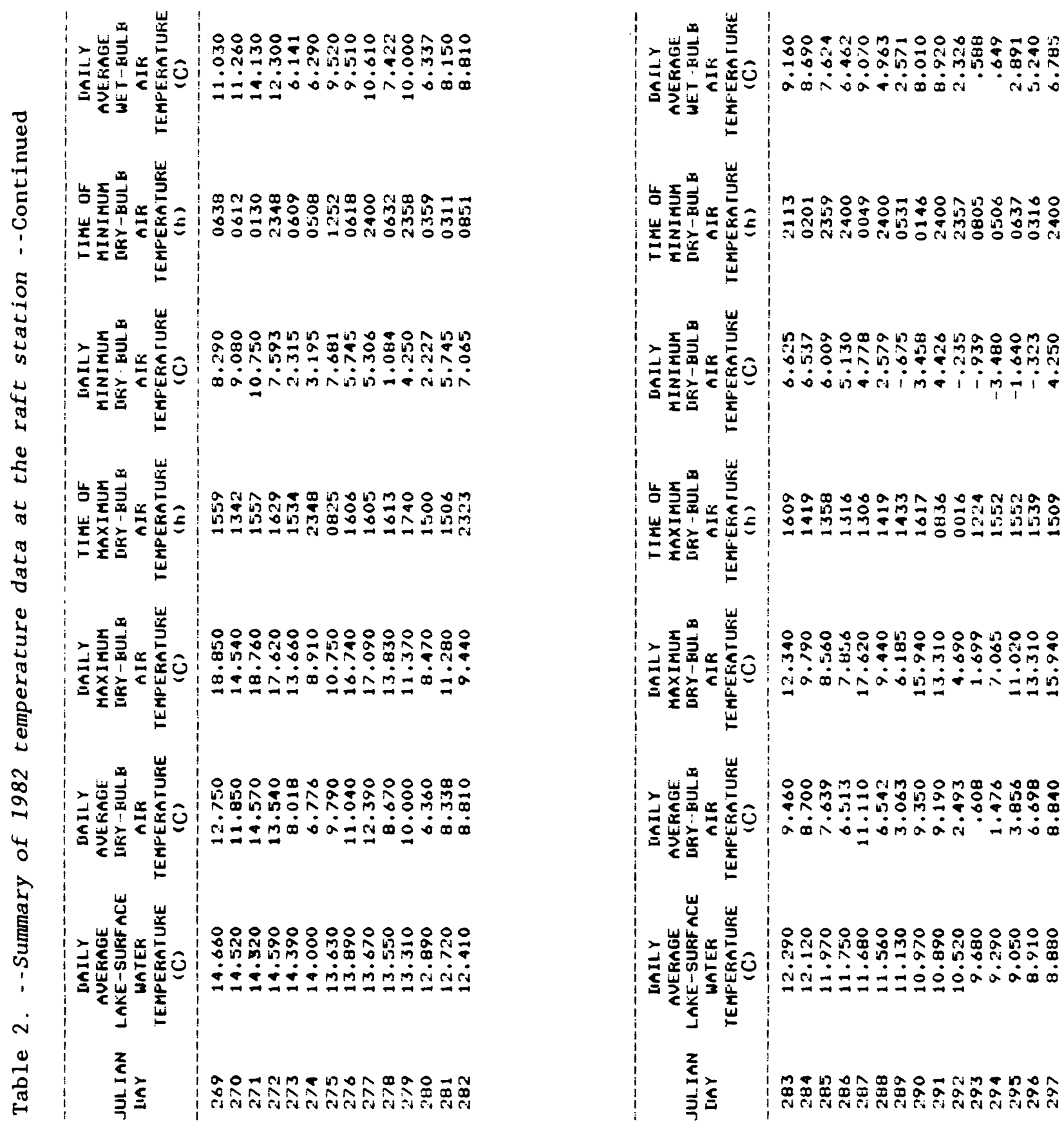
Table 3. --Summary of 1982 wind-speed data at the raft station [mi/h, miles per hour; h, hour; blank, no data]

\begin{tabular}{|c|c|c|c|c|c|c|}
\hline $\begin{array}{l}\text { HEIGHT OF } \\
\text { ANEMOMETEK } \\
\text { AEOUE LAKE } \\
\text { SURFACE } \\
\text { (m) }\end{array}$ & $\begin{array}{l}\text { JUL IAN } \\
\text { IIAY }\end{array}$ & $\begin{array}{l}\text { IIAILY } \\
\text { AUEFIAGE } \\
\text { WINEI } \\
\text { SFEEII } \\
(\mathrm{m} 1 / \mathrm{h})\end{array}$ & $\begin{array}{l}\text { IIATI.Y } \\
\text { MAXIMUM } \\
\text { WINII } \\
\text { SFEEU } \\
(m I / h)\end{array}$ & $\begin{array}{l}\text { TIME OF } \\
\text { MAXIMUM } \\
\text { WINII } \\
\text { SFEEI } \\
\text { (h) }\end{array}$ & $\begin{array}{l}\text { DAILY } \\
\text { MINIMUM } \\
\text { WIND } \\
\text { SFEEII } \\
(m 1 / h) 1\end{array}$ & $\begin{array}{l}\text { TIMF OF } \\
\text { MINIMUM } \\
\text { WINII } \\
\text { SFEEI } \\
(\mathrm{h})\end{array}$ \\
\hline 1 & $\begin{array}{l}164 \\
165 \\
166 \\
167 \\
168 \\
169 \\
170 \\
171 \\
172 \\
173 \\
174 \\
175 \\
176\end{array}$ & $\begin{array}{l}4.476 \\
2.722 \\
4.129 \\
3.455 \\
3.303 \\
1.798 \\
2.824 \\
4.791 \\
3.751 \\
2.103 \\
5.137 \\
5.626 \\
2.391\end{array}$ & 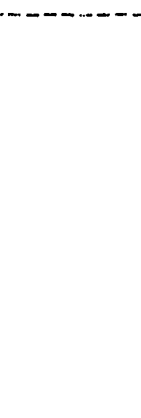 & $-\cdots---$ & ------ & $\rightarrow-\infty-\infty$ \\
\hline 2 & $\begin{array}{l}164 \\
165 \\
166 \\
167 \\
168 \\
169 \\
170 \\
171 \\
172 \\
173 \\
174 \\
175 \\
176\end{array}$ & $\begin{array}{l}5.234 \\
3.162 \\
4.696 \\
4.055 \\
3.713 \\
2.053 \\
3.306 \\
5.403 \\
4.166 \\
2.216 \\
5.898 \\
6.456 \\
2.473\end{array}$ & $\begin{array}{l}17.150 \\
16.330 \\
19.950 \\
18.710 \\
15.310 \\
14.600 \\
14.070 \\
17.660 \\
19.020 \\
15.540 \\
17.940 \\
19.730 \\
12.080\end{array}$ & $\begin{array}{l}1237 \\
1555 \\
1451 \\
1024 \\
1230 \\
1610 \\
1740 \\
1715 \\
1445 \\
1439 \\
1008 \\
1441 \\
0937\end{array}$ & $\begin{array}{r}0.600 \\
.600 \\
.600 \\
.571 \\
.571 \\
.571 \\
.600 \\
.600 \\
.600 \\
.571 \\
.571 \\
.600 \\
.571\end{array}$ & $\begin{array}{l}2057 \\
2035 \\
2344 \\
2005 \\
0022 \\
2027 \\
2337 \\
2353 \\
2400 \\
0321 \\
0315 \\
2318 \\
1356\end{array}$ \\
\hline 4 & $\begin{array}{l}164 \\
165 \\
166 \\
167 \\
168 \\
169 \\
170 \\
171 \\
172 \\
173 \\
174 \\
175 \\
176\end{array}$ & $\begin{array}{l}6.115 \\
3.502 \\
5.142 \\
4.627 \\
4.001 \\
2.190 \\
3.834 \\
6.362 \\
4.768 \\
2.491 \\
6.919 \\
7.572 \\
2.744\end{array}$ & $\begin{array}{l}19.100 \\
18.960 \\
21.250 \\
20.090 \\
15.140 \\
15.230 \\
15.510 \\
26.350 \\
18.710 \\
11.750 \\
19.190 \\
22.950 \\
11.430\end{array}$ & $\begin{array}{l}1158 \\
0248 \\
1451 \\
1003 \\
1230 \\
1610 \\
1450 \\
1543 \\
1445 \\
1439 \\
1521 \\
0159 \\
1600\end{array}$ & $\begin{array}{r}0.600 \\
.600 \\
.571 \\
.571 \\
.571 \\
.571 \\
.600 \\
.571 \\
.571 \\
.571 \\
.600 \\
.600 \\
.543\end{array}$ & $\begin{array}{l}2349 \\
2055 \\
2116 \\
2007 \\
0223 \\
2028 \\
2336 \\
2010 \\
2317 \\
0302 \\
0528 \\
2330 \\
1356\end{array}$ \\
\hline
\end{tabular}


Table 3. --Summary of 1982 wind-speed data at the raft station --Continued

\begin{tabular}{|c|c|c|c|c|c|c|}
\hline $\begin{array}{c}\text { HEIGHT OF } \\
\text { ANEMOMETER } \\
\text { AEQUE LAKE } \\
\text { SURFACE } \\
(m)\end{array}$ & $\begin{array}{l}\text { JUL IAN } \\
\text { IIAY }\end{array}$ & $\begin{array}{l}\text { IIAILY } \\
\text { AUERAGE } \\
\text { WINL } \\
\text { SFEEI } \\
(m i / h)\end{array}$ & $\begin{array}{l}\text { DAILY } \\
\text { MAXIMUM } \\
\text { WIND } \\
\text { SPEEI } \\
(m i / h)\end{array}$ & $\begin{array}{l}\text { TIME OF } \\
\text { MAXIMUM } \\
\text { WIND } \\
\text { SPEED } \\
\text { (h) }\end{array}$ & $\begin{array}{l}\text { DAILY } \\
\text { MINIMUM } \\
\text { WIND } \\
\text { SFEEII } \\
(\mathrm{mi} / \mathrm{h})\end{array}$ & $\begin{array}{l}\text { TIME OF } \\
\text { MINIMUH } \\
\text { WIND } \\
\text { SPEEII } \\
\text { (h) }\end{array}$ \\
\hline 1 & $\begin{array}{l}177 \\
178 \\
179 \\
180\end{array}$ & $\begin{array}{l}3.043 \\
2.903 \\
2.763 \\
2.674\end{array}$ & --- & $--\infty-$ & $=$ & ---- \\
\hline & 181 & $\therefore .159$ & 10.640 & 1023 & 0.600 & 2354 \\
\hline & $\begin{array}{l}182 \\
183\end{array}$ & $\begin{array}{l}3.666 \\
5.055\end{array}$ & $\begin{array}{l}12.000 \\
14.290\end{array}$ & $\begin{array}{l}1316 \\
1018\end{array}$ & $\begin{array}{r}.571 \\
.600\end{array}$ & $\begin{array}{l}0354 \\
2105\end{array}$ \\
\hline & $\begin{array}{l}184 \\
185 \\
186\end{array}$ & $\begin{array}{l}4.513 \\
4.546 \\
6.927\end{array}$ & $\begin{array}{l}20.630 \\
19.640 \\
23.180\end{array}$ & $\begin{array}{l}1358 \\
2245 \\
1419\end{array}$ & $\begin{array}{r}.600 \\
.600 \\
.600\end{array}$ & $\begin{array}{l}2354 \\
1009 \\
2249\end{array}$ \\
\hline & $\begin{array}{l}187 \\
188\end{array}$ & $\begin{array}{l}2.843 \\
6.544\end{array}$ & $\begin{array}{l}20.550 \\
23.940\end{array}$ & $\begin{array}{l}0002 \\
1250\end{array}$ & $\begin{array}{l}.600 \\
.600\end{array}$ & $\begin{array}{l}2216 \\
2342\end{array}$ \\
\hline 2 & $\begin{array}{l}177 \\
178 \\
179 \\
180 \\
181 \\
182 \\
183 \\
184 \\
185 \\
186 \\
187 \\
188\end{array}$ & $\begin{array}{l}3.485 \\
3.115 \\
2.909 \\
2.779 \\
2.113 \\
3.930 \\
5.596 \\
4.982 \\
5.027 \\
7.929 \\
2.967 \\
7.186\end{array}$ & $\begin{array}{l}14.040 \\
14.350 \\
20.800 \\
10.470 \\
15.340 \\
19.220 \\
21.930 \\
27.000 \\
28.500 \\
21.450 \\
24.450\end{array}$ & $\begin{array}{l}1152 \\
1459 \\
1115 \\
1023 \\
1323 \\
1205 \\
1345 \\
2300 \\
1539 \\
0009 \\
1250\end{array}$ & $\begin{array}{r}0.571 \\
.571 \\
.571 \\
.571 \\
.600 \\
.600 \\
.600 \\
.571 \\
.600 \\
.571 \\
.571\end{array}$ & $\begin{array}{l}0557 \\
2250 \\
2214 \\
2241 \\
2125 \\
2346 \\
2400 \\
0158 \\
2305 \\
0152 \\
2217\end{array}$ \\
\hline 4 & $\begin{array}{l}177 \\
178 \\
179 \\
180 \\
181 \\
182 \\
183 \\
184 \\
185 \\
186 \\
187 \\
188\end{array}$ & $\begin{array}{l}4.107 \\
3.696 \\
3.194 \\
2.984 \\
2.337 \\
4.746 \\
6.707 \\
6.051 \\
5.962 \\
9.210 \\
3.661 \\
8.330\end{array}$ & $\begin{array}{l}14.800 \\
14.800 \\
18.170 \\
12.590 \\
16.810 \\
19.410 \\
22.870 \\
28.390 \\
30.370 \\
21.370 \\
25.530\end{array}$ & $\begin{array}{l}1522 \\
1410 \\
1115 \\
1242 \\
1548 \\
1205 \\
1155 \\
2300 \\
1405 \\
0005 \\
0725\end{array}$ & $\begin{array}{r}0.571 \\
.571 \\
.571 \\
.571 \\
.571 \\
.600 \\
.600 \\
.600 \\
.600 \\
.571 \\
.600\end{array}$ & $\begin{array}{l}2029 \\
1922 \\
0604 \\
2241 \\
0038 \\
2313 \\
2351 \\
1338 \\
2251 \\
0152 \\
2349\end{array}$ \\
\hline
\end{tabular}


Table 3. --Summary of 1982 wind-speed data at the raft station --Continued

\begin{tabular}{|c|c|c|c|c|c|c|}
\hline $\begin{array}{l}\text { HE IGHT OF } \\
\text { ANEMOMETER } \\
\text { AEOUE LAKE } \\
\text { SURF ACE } \\
\text { (m) }\end{array}$ & $\begin{array}{l}\text { JUL IAN } \\
\text { DAY }\end{array}$ & $\begin{array}{l}\text { IIAILY } \\
\text { AUERAGE } \\
\text { WIND } \\
\text { SFEEII } \\
(\mathrm{mi} / \mathrm{h})\end{array}$ & $\begin{array}{l}\text { IAAILY } \\
\text { MAXIMUM } \\
\text { WINII } \\
\text { SFEEII } \\
(\mathrm{mi} / \mathrm{h})\end{array}$ & $\begin{array}{l}\text { TIME OF } \\
\text { MAXIMUM } \\
\text { WIND } \\
\text { SPEEII } \\
(h)\end{array}$ & $\begin{array}{l}\text { DAAILY } \\
\text { MINIMUA } \\
\text { WIND } \\
\text { SPEED } \\
(\mathrm{mi} / \mathrm{h})\end{array}$ & $\begin{array}{l}\text { TIME OF } \\
\text { MINIMUM } \\
\text { WINII } \\
\text { SFEEII } \\
\text { (h) }\end{array}$ \\
\hline 1 & $\begin{array}{l}189 \\
190 \\
191 \\
192 \\
193 \\
194 \\
195 \\
196 \\
197 \\
198 \\
199 \\
200 \\
201 \\
202 \\
203\end{array}$ & $\begin{array}{l}3.303 \\
3.219 \\
2.401 \\
3.250 \\
2.234 \\
1.919 \\
3.173 \\
4.802 \\
6.784 \\
3.441 \\
2.834 \\
2.585 \\
2.331\end{array}$ & $\begin{array}{r}13.100 \\
10.980 \\
9.990 \\
14.040 \\
14.380 \\
9.820 \\
12.820\end{array}$ & $\begin{array}{l}1201 \\
1431 \\
1026 \\
1039 \\
1617 \\
1236 \\
1029\end{array}$ & $\begin{array}{r}0.600 \\
.571 \\
.571 \\
.571 \\
.571 \\
.571 \\
.600\end{array}$ & $\begin{array}{l}2348 \\
0621 \\
1933 \\
0510 \\
0634 \\
2348 \\
2020\end{array}$ \\
\hline 2 & $\begin{array}{l}189 \\
190 \\
191 \\
192 \\
193 \\
194 \\
195 \\
196 \\
197 \\
198 \\
199 \\
200 \\
201 \\
202 \\
203\end{array}$ & $\begin{array}{l}3.492 \\
3.376 \\
2.373 \\
3.358 \\
2.152 \\
1.764 \\
3.406 \\
5.456 \\
7.741 \\
3.623 \\
2.887 \\
2.455 \\
2.310\end{array}$ & $\begin{array}{l}15.310 \\
11.010 \\
10.980 \\
15.030 \\
14.800 \\
10.130 \\
13.440\end{array}$ & $\begin{array}{l}1200 \\
1655 \\
1026 \\
1314 \\
1617 \\
1236 \\
1636\end{array}$ & $\begin{array}{r}0.571 \\
.571 \\
.571 \\
.571 \\
.571 \\
.571 \\
.600\end{array}$ & $\begin{array}{l}2119 \\
0625 \\
2355 \\
2345 \\
0638 \\
2353 \\
2319\end{array}$ \\
\hline 4 & $\begin{array}{l}189 \\
190 \\
191 \\
192 \\
193 \\
194 \\
195 \\
196 \\
197 \\
198 \\
199 \\
200 \\
201 \\
202 \\
203\end{array}$ & $\begin{array}{l}4.173 \\
3.900 \\
2.699 \\
4.098 \\
2.657 \\
2.174 \\
4.105 \\
\\
6.396 \\
9.220 \\
4.204 \\
3.669 \\
2.833 \\
2.703\end{array}$ & $\begin{array}{l}18.200 \\
13.020 \\
10.300 \\
15.230 \\
15.620 \\
10.420 \\
13.700\end{array}$ & $\begin{array}{l}1217 \\
1456 \\
1404 \\
1317 \\
1617 \\
1215 \\
1702\end{array}$ & $\begin{array}{l}0.571 \\
.571 \\
.571 \\
.600 \\
.571 \\
.571 \\
.571\end{array}$ & $\begin{array}{l}2119 \\
2334 \\
2354 \\
2400 \\
0635 \\
2348 \\
0510\end{array}$ \\
\hline
\end{tabular}


Table 3. --Summary of 1982 wind-speed data at the raft station --Continued

\begin{tabular}{|c|c|c|c|c|c|c|}
\hline $\begin{array}{l}\text { HEIGH OF } \\
\text { ANEMOME TER } \\
\text { AEOUE LAKE } \\
\text { SUFFACE } \\
(m)\end{array}$ & $\begin{array}{l}\text { JUL IAN } \\
\text { IIAY }\end{array}$ & $\begin{array}{l}\text { IIAILY } \\
\text { AUERAGE } \\
\text { WINII } \\
\text { SFEEII } \\
(\mathrm{m} 1 / \mathrm{h})\end{array}$ & $\begin{array}{l}\text { IAILY } \\
\text { MAXIMUM } \\
\text { WINII } \\
\text { SFEEII } \\
(\mathrm{mi} / \mathrm{h})\end{array}$ & $\begin{array}{l}\text { TIME OF } \\
\text { MAXIMUM } \\
\text { WINII } \\
\text { SFEEII } \\
\text { (h) }\end{array}$ & $\begin{array}{l}\text { IIAILY } \\
\text { MINIMUM } \\
\text { WIND } \\
\text { SFEEI } \\
\left(\mathrm{mI}_{1} / \mathrm{h}\right)\end{array}$ & $\begin{array}{l}\text { TIME OF } \\
\text { MINIMUM } \\
\text { WIND } \\
\text { SFEEII } \\
\text { (h) }\end{array}$ \\
\hline--------1 & $\begin{array}{l}204 \\
205 \\
206 \\
207 \\
208 \\
209 \\
210 \\
211 \\
212 \\
213 \\
214 \\
215 \\
216 \\
217\end{array}$ & $\begin{array}{l}5.729 \\
4.471 \\
2.222 \\
1.925 \\
1.522 \\
1.647 \\
5.775 \\
3.407 \\
2.808 \\
3.489 \\
6.180 \\
3.237 \\
2.780 \\
1.884\end{array}$ & $\begin{array}{r}18.060 \\
17.550 \\
9.740 \\
12.420 \\
8.410 \\
9.000 \\
19.270 \\
12.650 \\
11.830 \\
12.310 \\
19.080 \\
11.800 \\
12.740 \\
7.950\end{array}$ & $\begin{array}{l}1613 \\
0315 \\
1000 \\
1339 \\
1405 \\
1106 \\
1410 \\
1202 \\
1316 \\
1834 \\
1048 \\
1043 \\
0947 \\
1957\end{array}$ & $\begin{array}{r}0.883 \\
.600 \\
.571 \\
.515 \\
.401 \\
.600 \\
.600 \\
.571 \\
.600 \\
.571 \\
.741 \\
.600 \\
.600 \\
.571\end{array}$ & $\begin{array}{l}-142 \\
0142 \\
2349 \\
1743 \\
2235 \\
0631 \\
2319 \\
2231 \\
0519 \\
2400 \\
0156 \\
1504 \\
2400 \\
2400 \\
0723\end{array}$ \\
\hline 2 & $\begin{array}{l}204 \\
205 \\
206 \\
207 \\
208 \\
209 \\
210 \\
211 \\
212 \\
213 \\
214 \\
215 \\
216 \\
217\end{array}$ & $\begin{array}{l}6.536 \\
4.951 \\
2.166 \\
1.786 \\
1.520 \\
1.337 \\
6.500 \\
3.587 \\
3.002 \\
3.723 \\
6.979 \\
3.445 \\
2.774 \\
1.726\end{array}$ & $\begin{array}{r}16.900 \\
19.130 \\
12.110 \\
15.060 \\
10.250 \\
7.448 \\
24.450 \\
12.820 \\
15.680 \\
13.730 \\
20.910 \\
18.760 \\
13.330 \\
9.710\end{array}$ & $\begin{array}{l}1732 \\
0315 \\
0950 \\
1339 \\
1135 \\
1218 \\
1358 \\
1110 \\
1423 \\
2137 \\
1048 \\
0125 \\
0848 \\
1551\end{array}$ & $\begin{array}{l}0.600 \\
.600 \\
.571 \\
.571 \\
.571 \\
.571 \\
.600 \\
.571 \\
.571 \\
.571 \\
.600 \\
.571 \\
.571 \\
.571\end{array}$ & $\begin{array}{l}0142 \\
2400 \\
2113 \\
2317 \\
2204 \\
0642 \\
2357 \\
2046 \\
2221 \\
0720 \\
1504 \\
2025 \\
1746 \\
1913\end{array}$ \\
\hline 4 & $\begin{array}{l}204 \\
205 \\
206 \\
207 \\
208 \\
209 \\
210 \\
211 \\
212 \\
213 \\
214 \\
215 \\
216 \\
217\end{array}$ & $\begin{array}{l}7.687 \\
5.900 \\
2.666 \\
2.216 \\
1.962 \\
1.825 \\
7.616 \\
4.290 \\
3.484 \\
4.292 \\
8.190 \\
4.197 \\
3.357 \\
2.150\end{array}$ & $\begin{array}{r}20.010 \\
20.210 \\
13.300 \\
14.120 \\
12.760 \\
9.790 \\
23.520 \\
13.780 \\
15.680 \\
15.510 \\
20.520 \\
21.880 \\
13.390 \\
11.410\end{array}$ & $\begin{array}{l}1732 \\
0535 \\
0950 \\
1339 \\
1431 \\
1106 \\
1358 \\
1132 \\
1423 \\
2341 \\
1048 \\
0125 \\
0944 \\
1053\end{array}$ & $\begin{array}{r}0.628 \\
.600 \\
.600 \\
.571 \\
.571 \\
.571 \\
.600 \\
.571 \\
.571 \\
.288 \\
2.071 \\
.571 \\
.571 \\
.571\end{array}$ & $\begin{array}{l}0138 \\
2353 \\
2400 \\
2314 \\
2204 \\
1423 \\
2233 \\
2046 \\
2221 \\
0810 \\
1504 \\
2218 \\
2033 \\
1913\end{array}$ \\
\hline
\end{tabular}


Table 3. --Sumary of 1982 wind-speed data at the raft station --Continued

\begin{tabular}{|c|c|c|c|c|c|c|}
\hline $\begin{array}{l}\text { HE IGHT OF } \\
\text { ANEMOME IEE } \\
\text { AEOUE LAKE } \\
\text { SURF ACE } \\
(m)\end{array}$ & $\begin{array}{l}\text { JUL IAN } \\
\text { IIAY }\end{array}$ & $\begin{array}{l}\text { DAILY } \\
\text { AUEFAGE. } \\
\text { WINI! } \\
\text { SFEEII } \\
(m i / h)\end{array}$ & $\begin{array}{l}\text { DAILY } \\
\text { MAXIMUM } \\
\text { WIND } \\
\text { SFEEII } \\
(m i / h)\end{array}$ & $\begin{array}{l}\text { YIME OF } \\
\text { MAXIMUM } \\
\text { WIND } \\
\text { SFEEII } \\
\text { (h) }\end{array}$ & $\begin{array}{l}\text { IIAILY } \\
\text { MINIMUM } \\
\text { WIND } \\
\text { SFEEII } \\
(m i / h)\end{array}$ & $\begin{array}{l}\text { TIME OF } \\
\text { MINIMUM } \\
\text { WINI } \\
\text { SFEEII } \\
\text { (h) }\end{array}$ \\
\hline 1 & $\begin{array}{l}218 \\
219 \\
220 \\
221 \\
222 \\
223 \\
224 \\
225 \\
226 \\
227 \\
228 \\
229\end{array}$ & $\begin{array}{l}2.605 \\
4.535 \\
7.770 \\
5.487 \\
2.582 \\
3.530 \\
6.342 \\
3.307 \\
4.171 \\
2.970 \\
1.650 \\
2.269\end{array}$ & $\begin{array}{l}14.630 \\
17.150 \\
21.570 \\
18.930 \\
11.070 \\
12.960 \\
25.670 \\
13.080 \\
11.660 \\
13.130 \\
13.100 \\
9.090\end{array}$ & $\begin{array}{l}0701 \\
1710 \\
1302 \\
1247 \\
1137 \\
1146 \\
1021 \\
0104 \\
1323 \\
1024 \\
1040 \\
1449\end{array}$ & $\begin{array}{r}0.600 \\
.600 \\
1.449 \\
.600 \\
.600 \\
.600 \\
.600 \\
.600 \\
.600 \\
.600 \\
.571 \\
.571\end{array}$ & $\begin{array}{l}2304 \\
1156 \\
1850 \\
2353 \\
2400 \\
0742 \\
1907 \\
1650 \\
2100 \\
2354 \\
0634 \\
2237\end{array}$ \\
\hline 2 & $\begin{array}{l}218 \\
219 \\
220 \\
221 \\
222 \\
223 \\
224 \\
225 \\
226 \\
227 \\
228 \\
229\end{array}$ & $\begin{array}{l}2.619 \\
5.103 \\
8.750 \\
6.091 \\
2.413 \\
3.701 \\
6.905 \\
3.595 \\
4.712 \\
3.032 \\
1.429 \\
2.173\end{array}$ & $\begin{array}{l}14.430 \\
17.520 \\
22.390 \\
21.480 \\
13.190 \\
15.170 \\
21.990 \\
11.580 \\
16.410 \\
12.820 \\
13.190 \\
9.230\end{array}$ & $\begin{array}{l}1109 \\
1710 \\
1305 \\
1159 \\
1055 \\
1357 \\
1110 \\
0104 \\
1314 \\
1024 \\
1036 \\
1414\end{array}$ & $\begin{array}{r}0.571 \\
.571 \\
.684 \\
.571 \\
.571 \\
.571 \\
.600 \\
.600 \\
.600 \\
.571 \\
.571 \\
.571\end{array}$ & $\begin{array}{l}2203 \\
0242 \\
1851 \\
2107 \\
2249 \\
0655 \\
1934 \\
1650 \\
2329 \\
2027 \\
1702 \\
2237\end{array}$ \\
\hline 4 & $\begin{array}{l}218 \\
219 \\
220 \\
221 \\
222 \\
223 \\
224 \\
225 \\
226 \\
227 \\
228 \\
229\end{array}$ & $\begin{array}{r}3.268 \\
6.036 \\
10.370 \\
7.081 \\
2.994 \\
4.454 \\
8.030 \\
4.309 \\
5.539 \\
3.830 \\
1.909 \\
2.829\end{array}$ & $\begin{array}{r}15.540 \\
24.110 \\
26.570 \\
20.660 \\
14.120 \\
16.190 \\
26.090 \\
12.820 \\
15.910 \\
17.150 \\
14.150 \\
9.620\end{array}$ & $\begin{array}{l}1112 \\
1710 \\
1701 \\
1249 \\
1252 \\
1554 \\
1021 \\
0104 \\
1314 \\
1024 \\
1034 \\
1410\end{array}$ & $\begin{array}{r}0.571 \\
.571 \\
1.816 \\
.571 \\
.571 \\
.571 \\
.600 \\
.458 \\
.600 \\
.571 \\
.571 \\
.543\end{array}$ & $\begin{array}{l}2203 \\
0238 \\
1852 \\
2106 \\
2249 \\
0654 \\
1907 \\
1700 \\
2105 \\
2027 \\
1422 \\
1150\end{array}$ \\
\hline
\end{tabular}


Table 3. --Summary of 1982 wind-speed data at the raft station --Continued

\begin{tabular}{|c|c|c|c|c|c|c|}
\hline $\begin{array}{l}\text { HE IGHT OF } \\
\text { ANEMOME TEF } \\
\text { ABOUE LAKE } \\
\text { SURF ACE } \\
(\mathrm{m})\end{array}$ & $\begin{array}{l}\text { JUL IAN } \\
\text { IIAY }\end{array}$ & $\begin{array}{l}\text { JAILY } \\
\text { AUERAGE. } \\
\text { WINII } \\
\text { SFEEII } \\
(m 1 / h)\end{array}$ & $\begin{array}{l}\text { IIAILY } \\
\text { MAXIMUM } \\
\text { WIND } \\
\text { SFEED } \\
(m i / h)\end{array}$ & $\begin{array}{l}\text { TIME OF } \\
\text { MAXIMUH } \\
\text { WINI } \\
\text { SFEEII } \\
\text { (h) }\end{array}$ & $\begin{array}{l}\text { IIAILY } \\
\text { MINIMUM } \\
\text { WINII } \\
\text { SPEEII } \\
(m i / h)\end{array}$ & $\begin{array}{l}\text { TIME OF } \\
\text { MINIMUM } \\
\text { WINII } \\
\text { SFEEI } \\
\text { (h) }\end{array}$ \\
\hline 1 & $\begin{array}{l}230 \\
231 \\
232 \\
233 \\
234 \\
235 \\
236 \\
237 \\
238 \\
239\end{array}$ & $\begin{array}{l}4.332 \\
2.955 \\
3.339 \\
3.721 \\
3.779 \\
2.883 \\
3.541 \\
4.815 \\
2.911 \\
3.216\end{array}$ & $\begin{array}{l}19.700 \\
10.980 \\
15.620 \\
18.760 \\
12.370 \\
11.660 \\
21.740 \\
16.870 \\
12.480 \\
14.460\end{array}$ & $\begin{array}{l}1925 \\
0807 \\
1205 \\
1922 \\
0815 \\
1123 \\
1523 \\
1241 \\
1839 \\
1605\end{array}$ & $\begin{array}{r}0.600 \\
.600 \\
.571 \\
.600 \\
.600 \\
.571 \\
.600 \\
.600 \\
.600 \\
.600\end{array}$ & $\begin{array}{l}2025 \\
2344 \\
0043 \\
2351 \\
2351 \\
2311 \\
2334 \\
1905 \\
2347 \\
2356\end{array}$ \\
\hline 2 & $\begin{array}{l}230 \\
231 \\
232 \\
233 \\
234 \\
235 \\
236 \\
237 \\
238 \\
239\end{array}$ & $\begin{array}{l}4.946 \\
3.029 \\
3.561 \\
4.054 \\
4.147 \\
2.903 \\
3.627 \\
5.314 \\
2.849 \\
3.132\end{array}$ & $\begin{array}{l}26.210 \\
12.620 \\
18.820 \\
22.640 \\
14.600 \\
13.270 \\
23.010 \\
22.980 \\
15.880 \\
14.890\end{array}$ & $\begin{array}{l}1945 \\
1534 \\
1109 \\
1944 \\
0815 \\
0758 \\
1523 \\
1200 \\
1714 \\
1104\end{array}$ & $\begin{array}{r}0.600 \\
.600 \\
.571 \\
.571 \\
.571 \\
.571 \\
.571 \\
.571 \\
.571 \\
.571\end{array}$ & $\begin{array}{l}2025 \\
2400 \\
2146 \\
0043 \\
1924 \\
2315 \\
2144 \\
0005 \\
1300 \\
1843\end{array}$ \\
\hline 4 & $\begin{array}{l}230 \\
231 \\
232 \\
233 \\
234 \\
235 \\
236 \\
237 \\
238 \\
239\end{array}$ & $\begin{array}{l}5.840 \\
3.591 \\
4.211 \\
4.754 \\
4.711 \\
3.316 \\
4.332 \\
6.181 \\
3.429 \\
3.801\end{array}$ & $\begin{array}{l}26.770 \\
14.430 \\
19.100 \\
21.280 \\
14.460 \\
13.050 \\
20.550 \\
19.190 \\
16.100 \\
15.170\end{array}$ & $\begin{array}{l}1945 \\
1349 \\
1138 \\
1944 \\
1406 \\
1037 \\
1553 \\
1200 \\
1839 \\
1221\end{array}$ & $\begin{array}{r}0.600 \\
.600 \\
.600 \\
.571 \\
.600 \\
.571 \\
.571 \\
.571 \\
.600 \\
.571\end{array}$ & $\begin{array}{l}1900 \\
2352 \\
2400 \\
0036 \\
2351 \\
2312 \\
0617 \\
0003 \\
2354 \\
0504\end{array}$ \\
\hline
\end{tabular}


Table 3. --Summary of 1982 wind-speed data at the raft station --Continued

\begin{tabular}{|c|c|c|c|c|c|c|}
\hline $\begin{array}{l}\text { HEIGHT OF } \\
\text { ANEMOKETEE } \\
\text { ABOUE LAKE } \\
\text { SUFIF ACE } \\
(\mathrm{m})\end{array}$ & $\begin{array}{l}\text { JUL I AN } \\
\text { IIAY }\end{array}$ & $\begin{array}{l}\text { [IAILY } \\
\text { AUEFAGE } \\
\text { WINII } \\
\text { SFEED } \\
(m i / h)\end{array}$ & $\begin{array}{l}\text { IIAILY } \\
\text { MAXIMUM } \\
\text { HINI } \\
\text { SFEEI } \\
(m i / h)\end{array}$ & $\begin{array}{l}\text { TIME OF } \\
\text { MAXIMUH } \\
\text { WIND } \\
\text { SFEED } \\
\text { (h) }\end{array}$ & $\begin{array}{l}\text { UAILY } \\
\text { MINIMUM } \\
\text { WIND } \\
\text { SFEEI } \\
(\mathrm{mI} / \mathrm{h})\end{array}$ & $\begin{array}{l}\text { TIME OF } \\
\text { MINIMUM } \\
\text { WINI } \\
\text { SFEED } \\
\text { (h) }\end{array}$ \\
\hline 1 & $\begin{array}{l}240 \\
241 \\
242 \\
243 \\
244 \\
245 \\
246 \\
247 \\
248 \\
249 \\
250 \\
251 \\
252 \\
253 \\
254 \\
255\end{array}$ & $\begin{array}{l}5.178 \\
3.278 \\
2.670 \\
3.092 \\
4.451 \\
5.067 \\
2.617 \\
4.377 \\
3.863 \\
4.062 \\
5.548 \\
7.190 \\
6.276 \\
3.692 \\
5.031 \\
4.413\end{array}$ & $\begin{array}{r}15.080 \\
12.760 \\
9.250 \\
8.750 \\
19.950 \\
18.310 \\
12.030 \\
18.400 \\
14.150 \\
12.450 \\
13.980 \\
19.920 \\
16.130 \\
11.120 \\
16.900 \\
17.660\end{array}$ & $\begin{array}{l}2322 \\
1503 \\
1454 \\
0700 \\
2148 \\
1343 \\
1006 \\
1006 \\
0906 \\
1144 \\
1734 \\
1220 \\
1319 \\
1200 \\
1213 \\
1732\end{array}$ & $\begin{array}{r}0.600 \\
.600 \\
.571 \\
.600 \\
.600 \\
.600 \\
.571 \\
.571 \\
.600 \\
.600 \\
1.335 \\
1.477 \\
.939 \\
.600 \\
.600 \\
.600\end{array}$ & $\begin{array}{l}0335 \\
2359 \\
1743 \\
2338 \\
1038 \\
2348 \\
2129 \\
1903 \\
2400 \\
1317 \\
0603 \\
2351 \\
1433 \\
1837 \\
2400 \\
1300\end{array}$ \\
\hline 2 & $\begin{array}{l}240 \\
241 \\
243 \\
243 \\
244 \\
245 \\
246 \\
247 \\
248 \\
249 \\
250 \\
251 \\
252 \\
253 \\
254 \\
255\end{array}$ & $\begin{array}{l}5.787 \\
3.443 \\
2.830 \\
3.665 \\
5.125 \\
5.783 \\
3.112 \\
5.278 \\
4.437 \\
4.538 \\
6.207 \\
8.050 \\
7.107 \\
4.109 \\
5.510 \\
4.840\end{array}$ & $\begin{array}{l}17.690 \\
13.390 \\
10.900 \\
10.470 \\
21.990 \\
20.720 \\
13.250 \\
22.640 \\
16.730 \\
14.260 \\
15.850 \\
26.660 \\
18.060 \\
14.460 \\
18.570 \\
18.250\end{array}$ & $\begin{array}{l}2302 \\
1540 \\
1252 \\
0639 \\
2129 \\
1554 \\
1116 \\
1334 \\
0906 \\
1444 \\
2134 \\
1309 \\
1231 \\
0033 \\
1104 \\
1702\end{array}$ & $\begin{array}{r}0.600 \\
.571 \\
.571 \\
.600 \\
.600 \\
.600 \\
.600 \\
.571 \\
.600 \\
.684 \\
1.930 \\
2.184 \\
2.241 \\
.600 \\
.600 \\
.600\end{array}$ & $\begin{array}{l}1427 \\
2154 \\
0727 \\
2333 \\
0956 \\
2345 \\
2246 \\
1903 \\
2153 \\
1240 \\
0543 \\
1834 \\
1404 \\
1036 \\
0822 \\
1238\end{array}$ \\
\hline 4 & $\begin{array}{l}240 \\
241 \\
2.42 \\
243 \\
2.44 \\
245 \\
246 \\
247 \\
248 \\
249 \\
250 \\
251 \\
252 \\
253 \\
254 \\
255\end{array}$ & $\begin{array}{l}6.911 \\
4.232 \\
3.152 \\
3.966 \\
5.797 \\
6.462 \\
3.105 \\
5.551 \\
4.429 \\
4.926 \\
7.366 \\
9.440 \\
8.450 \\
4.927 \\
6.548 \\
5.330\end{array}$ & $\begin{array}{l}20.550 \\
14.380 \\
11.890 \\
11.010 \\
20.580 \\
22.610 \\
15.990 \\
19.440 \\
17.410 \\
17.260 \\
16.870 \\
23.660 \\
21.340 \\
13.750 \\
18.400 \\
15.840\end{array}$ & $\begin{array}{l}2322 \\
1500 \\
1252 \\
1057 \\
2148 \\
1007 \\
1050 \\
1412 \\
0822 \\
0830 \\
2152 \\
1309 \\
1319 \\
1200 \\
1213 \\
1646\end{array}$ & $\begin{array}{r}0.600 \\
.5 / 1 \\
.571 \\
.600 \\
.600 \\
.571 \\
.571 \\
.571 \\
.571 \\
.600 \\
1.845 \\
1.618 \\
2.156 \\
.600 \\
.600 \\
.571\end{array}$ & $\begin{array}{l}0144 \\
1200 \\
1744 \\
2339 \\
1642 \\
2230 \\
2130 \\
1903 \\
1808 \\
1609 \\
1537 \\
0719 \\
1500 \\
1829 \\
2400 \\
1256\end{array}$ \\
\hline
\end{tabular}


Table 3. --Summary of 1982 wind-speed data at the raft station -Continued

\begin{tabular}{|c|c|c|c|c|c|c|}
\hline $\begin{array}{l}\text { HEIGHT OF } \\
\text { ANEMOMETEF } \\
\text { ABOUE LAKE } \\
\text { SUFFACE } \\
(m)\end{array}$ & $\underset{\text { JUAY }}{\text { JUL I AN }}$ & $\begin{array}{l}\text { IUAILY } \\
\text { AUERAGE } \\
\text { WINII } \\
\text { SFEEI } \\
(m 1 / n)\end{array}$ & $\begin{array}{l}\text { IIAII_Y } \\
\text { MAXIMUM } \\
\text { WINII } \\
\text { SFEEII } \\
\text { (mI/h) }\end{array}$ & $\begin{array}{l}\text { TIME OF } \\
\text { MAXIMUM } \\
\text { WINII } \\
\text { SPEEII } \\
\text { (h) }\end{array}$ & $\begin{array}{l}\text { DAILYY } \\
\text { MINIMUM } \\
\text { WINI } \\
\text { SFEEI } \\
(\mathrm{mi} / \mathrm{h})\end{array}$ & $\begin{array}{l}\text { TIME OF } \\
\text { MINIMUM } \\
\text { WINI! } \\
\text { SFEEII } \\
\text { (H) }\end{array}$ \\
\hline 1 & $\begin{array}{l}256 \\
257 \\
258 \\
259 \\
260 \\
261 \\
262 \\
263 \\
264 \\
265 \\
266 \\
267 \\
268\end{array}$ & $\begin{array}{l}3.879 \\
3.903 \\
2.276 \\
4.143 \\
4.283 \\
4.165 \\
5.393 \\
3.092 \\
1.578 \\
6.017 \\
5.235 \\
5.179 \\
3.637\end{array}$ & $\begin{array}{r}16.920 \\
12.880 \\
9.000 \\
13.730 \\
14.940 \\
17.040 \\
19.160 \\
16.730 \\
6.967 \\
19.810 \\
14.260 \\
14.350 \\
13.560\end{array}$ & $\begin{array}{l}1320 \\
1520 \\
1353 \\
1413 \\
1533 \\
1333 \\
0958 \\
1349 \\
1417 \\
1349 \\
2242 \\
1049 \\
1334\end{array}$ & $\begin{array}{r}0.600 \\
.600 \\
.600 \\
.600 \\
.600 \\
.600 \\
.600 \\
.571 \\
.571 \\
.600 \\
.600 \\
.600 \\
.600\end{array}$ & $\begin{array}{l}2316 \\
2347 \\
2358 \\
0708 \\
2359 \\
2400 \\
2359 \\
2321 \\
2046 \\
0121 \\
0704 \\
2352 \\
0928\end{array}$ \\
\hline 2 & $\begin{array}{l}256 \\
257 \\
258 \\
259 \\
260 \\
261 \\
262 \\
263 \\
264 \\
265 \\
266 \\
267 \\
268\end{array}$ & $\begin{array}{l}4.221 \\
4.252 \\
2.561 \\
4.596 \\
4.685 \\
4.639 \\
6.039 \\
3.464 \\
1.847 \\
6.726 \\
5.892 \\
5.813 \\
4.188\end{array}$ & $\begin{array}{r}17.320 \\
15.310 \\
10.250 \\
14.210 \\
14.580 \\
18.510 \\
17.240 \\
16.780 \\
7.950 \\
21.230 \\
16.440 \\
17.860 \\
14.120\end{array}$ & $\begin{array}{l}1320 \\
1227 \\
1210 \\
1352 \\
1533 \\
1446 \\
1241 \\
1036 \\
1412 \\
1234 \\
2148 \\
0112 \\
1334\end{array}$ & $\begin{array}{r}0.769 \\
.600 \\
.600 \\
.600 \\
.600 \\
.600 \\
.600 \\
.600 \\
.571 \\
.600 \\
.600 \\
.600 \\
.600\end{array}$ & $\begin{array}{l}2213 \\
1630 \\
2344 \\
0638 \\
2311 \\
2400 \\
0103 \\
2338 \\
1332 \\
0105 \\
0704 \\
2352 \\
0925\end{array}$ \\
\hline 4 & $\begin{array}{l}256 \\
257 \\
258 \\
259 \\
260 \\
261 \\
262 \\
263 \\
264 \\
265 \\
266 \\
267 \\
268\end{array}$ & $\begin{array}{l}4.621 \\
4.598 \\
2.509 \\
5.225 \\
5.473 \\
5.225 \\
6.916 \\
3.605 \\
1.690 \\
7.875 \\
6.798 \\
6.316 \\
4.479\end{array}$ & $\begin{array}{r}17.890 \\
16.640 \\
11.290 \\
15.820 \\
16.560 \\
21.740 \\
18.310 \\
17.010 \\
7.950 \\
24.540 \\
16.580 \\
19.560 \\
16.440\end{array}$ & $\begin{array}{l}1320 \\
1227 \\
1208 \\
1446 \\
1317 \\
1322 \\
1952 \\
1034 \\
1412 \\
1348 \\
2220 \\
1426 \\
1409\end{array}$ & $\begin{array}{r}0.600 \\
.571 \\
.571 \\
.571 \\
.600 \\
.571 \\
.600 \\
.571 \\
.571 \\
.600 \\
.600 \\
.600 \\
.571\end{array}$ & $\begin{array}{l}2321 \\
1548 \\
2358 \\
0144 \\
2259 \\
0037 \\
2400 \\
2321 \\
2247 \\
0122 \\
0704 \\
2358 \\
0414\end{array}$ \\
\hline
\end{tabular}


Table 3. --Summary of 1982 wind-speed data at the raft station --Continued

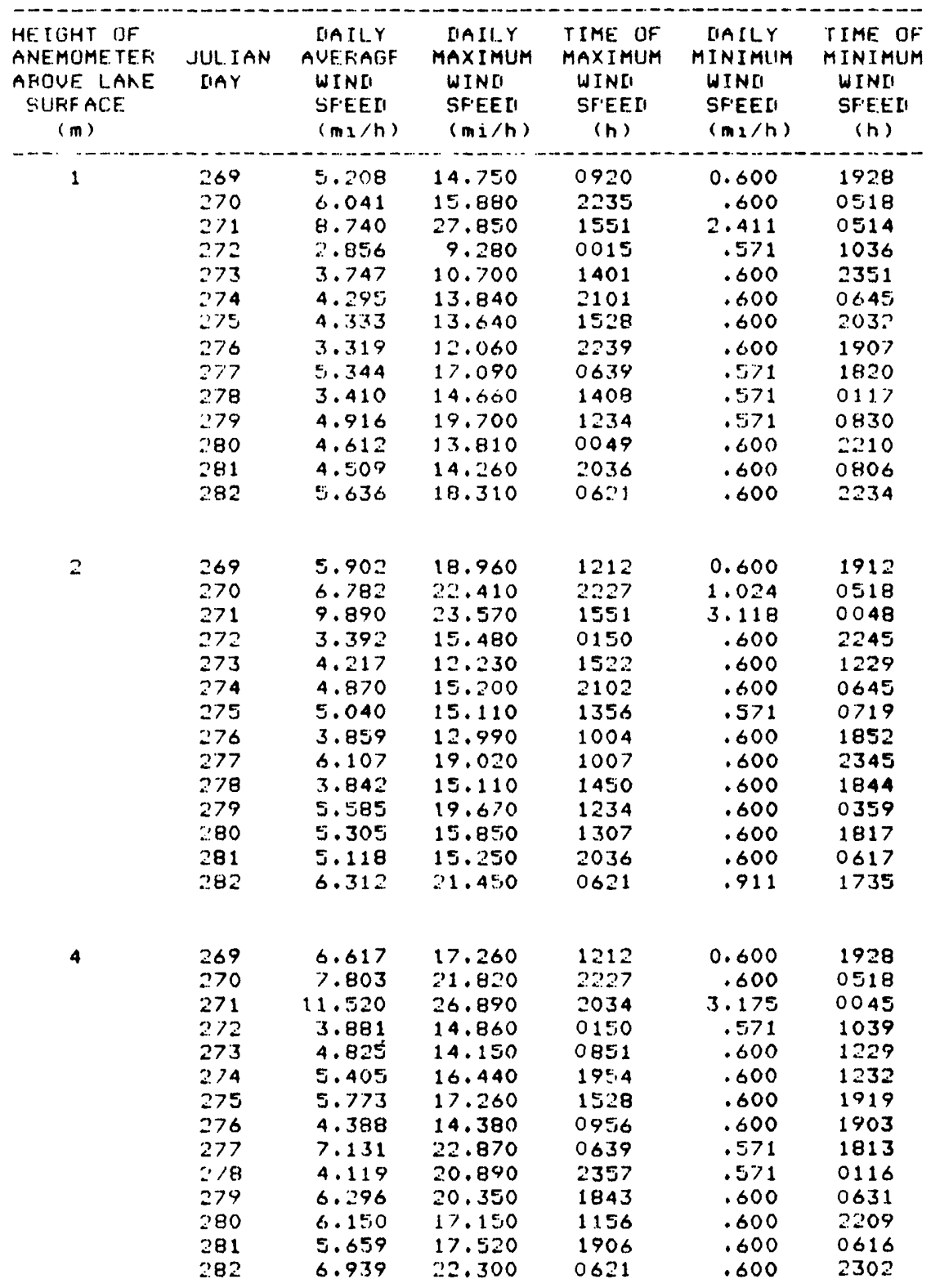


Table 3. --Summary of 1982 wind-speed data at the raft station --Continued

\begin{tabular}{|c|c|c|c|c|c|c|}
\hline $\begin{array}{l}\text { HEIGH T OF } \\
\text { ANE.MOME.TER } \\
\text { ABUUE LAKE } \\
\text { SURF AI:E } \\
(\mathrm{m})\end{array}$ & $\begin{array}{l}\text { JUL I AN } \\
\text { IIAY }\end{array}$ & $\begin{array}{l}\text { LIAII.Y } \\
\text { AUE.KAGE } \\
\text { WINII } \\
\text { SFEEII } \\
(m i / h)\end{array}$ & $\begin{array}{l}\text { IIAILY } \\
\text { MAXIMUM } \\
\text { WINI } \\
\text { SFEEII } \\
(\mathrm{mi} / \mathrm{h})\end{array}$ & $\begin{array}{l}\text { TIME OF } \\
\text { MAXIMUM } \\
\text { WINII } \\
\text { SFEEII } \\
\text { (h) }\end{array}$ & $\begin{array}{l}\text { IIAILY } \\
\text { MINIMUM } \\
\text { WINII } \\
\text { SFEEII } \\
(\mathrm{mI} / \mathrm{h})\end{array}$ & $\begin{array}{l}\text { TIME OF } \\
\text { MINIMUM } \\
\text { WINII } \\
\text { SFEEII } \\
\text { (h) }\end{array}$ \\
\hline 1 & $\begin{array}{l}283 \\
284 \\
285 \\
286 \\
287 \\
288 \\
289 \\
290 \\
291 \\
292 \\
293 \\
294 \\
295 \\
296 \\
297\end{array}$ & $\begin{array}{l}1.971 \\
3.296 \\
4.283 \\
4.147 \\
5.026 \\
6.524 \\
2.781 \\
5.486 \\
4.128 \\
6.060 \\
7.089 \\
2.596 \\
3.414 \\
3.971 \\
4.377\end{array}$ & $\begin{array}{r}6.684 \\
9.140 \\
13.470 \\
13.440 \\
25.240 \\
20.260 \\
10.020 \\
15.850 \\
14.430 \\
18.140 \\
19.410 \\
10.950 \\
10.980 \\
14.320 \\
14.010\end{array}$ & $\begin{array}{l}1645 \\
1123 \\
1213 \\
1410 \\
1153 \\
0858 \\
2154 \\
0503 \\
1321 \\
2115 \\
1145 \\
1427 \\
1425 \\
1527 \\
1350\end{array}$ & $\begin{array}{l}0.600 \\
.600 \\
.826 \\
.600 \\
.571 \\
.600 \\
.600 \\
.600 \\
.600 \\
1.279 \\
1.703 \\
.571 \\
.600 \\
.600 \\
.600\end{array}$ & $\begin{array}{l}2400 \\
1821 \\
2008 \\
2029 \\
2117 \\
0252 \\
1731 \\
1727 \\
1115 \\
0130 \\
2318 \\
0846 \\
2334 \\
1849 \\
2400\end{array}$ \\
\hline$?$ & $\begin{array}{l}283 \\
284 \\
285 \\
286 \\
287 \\
288 \\
289 \\
290 \\
291 \\
292 \\
293 \\
294 \\
295 \\
296 \\
297\end{array}$ & $\begin{array}{l}2.403 \\
3.765 \\
4.952 \\
4.775 \\
5.769 \\
7.471 \\
3.231 \\
6.299 \\
4.765 \\
6.853 \\
8.070 \\
3.068 \\
3.922 \\
4.619 \\
5.086\end{array}$ & $\begin{array}{r}1.646 \\
9.480 \\
13.360 \\
12.080 \\
26.010 \\
23.490 \\
11.090 \\
19.700 \\
14.460 \\
17.830 \\
19.500 \\
10.900 \\
10.220 \\
13.700 \\
14.490\end{array}$ & $\begin{array}{l}1306 \\
2258 \\
1314 \\
1454 \\
1153 \\
1202 \\
2324 \\
0322 \\
1632 \\
1841 \\
1145 \\
1427 \\
1247 \\
1527 \\
1350\end{array}$ & $\begin{array}{r}0.600 \\
.600 \\
1.364 \\
.939 \\
.600 \\
.600 \\
.600 \\
1.024 \\
.600 \\
1.618 \\
1.873 \\
.600 \\
.600 \\
.600 \\
.571\end{array}$ & $\begin{array}{l}2400 \\
0002 \\
2008 \\
0024 \\
2352 \\
0252 \\
1419 \\
1721 \\
1115 \\
0837 \\
1644 \\
1905 \\
2329 \\
1839 \\
2329\end{array}$ \\
\hline 4 & $\begin{array}{l}283 \\
284 \\
285 \\
286 \\
287 \\
288 \\
289 \\
290 \\
291 \\
292 \\
293 \\
294 \\
295 \\
296 \\
297\end{array}$ & $\begin{array}{l}2.413 \\
4.327 \\
5.694 \\
5.594 \\
6.801 \\
8.600 \\
3.310 \\
7.329 \\
5.239 \\
7.636 \\
9.290 \\
3.203 \\
4.423 \\
5.242 \\
5.667\end{array}$ & $\begin{array}{r}8.460 \\
10.560 \\
13.640 \\
13.920 \\
28.900 \\
25.810 \\
12.450 \\
20.800 \\
15.280 \\
20.040 \\
21.200 \\
12.200 \\
11.350 \\
15.170 \\
16.220\end{array}$ & $\begin{array}{l}0223 \\
2029 \\
1314 \\
1458 \\
1425 \\
1501 \\
2324 \\
0514 \\
0759 \\
1806 \\
1117 \\
1304 \\
1133 \\
1554 \\
1353\end{array}$ & $\begin{array}{r}0.600 \\
.600 \\
1.052 \\
.600 \\
.600 \\
.600 \\
.571 \\
.996 \\
.600 \\
.600 \\
.600 \\
.600 \\
.600 \\
.571 \\
.571\end{array}$ & $\begin{array}{l}2400 \\
1806 \\
2008 \\
1241 \\
2400 \\
2355 \\
1108 \\
1731 \\
1115 \\
0837 \\
1644 \\
2012 \\
2359 \\
0335 \\
2348\end{array}$ \\
\hline
\end{tabular}

1 Values of 0.600 or 0.571 are threshold values that represent the minimum windspeed required to turn the anemometer cups. 

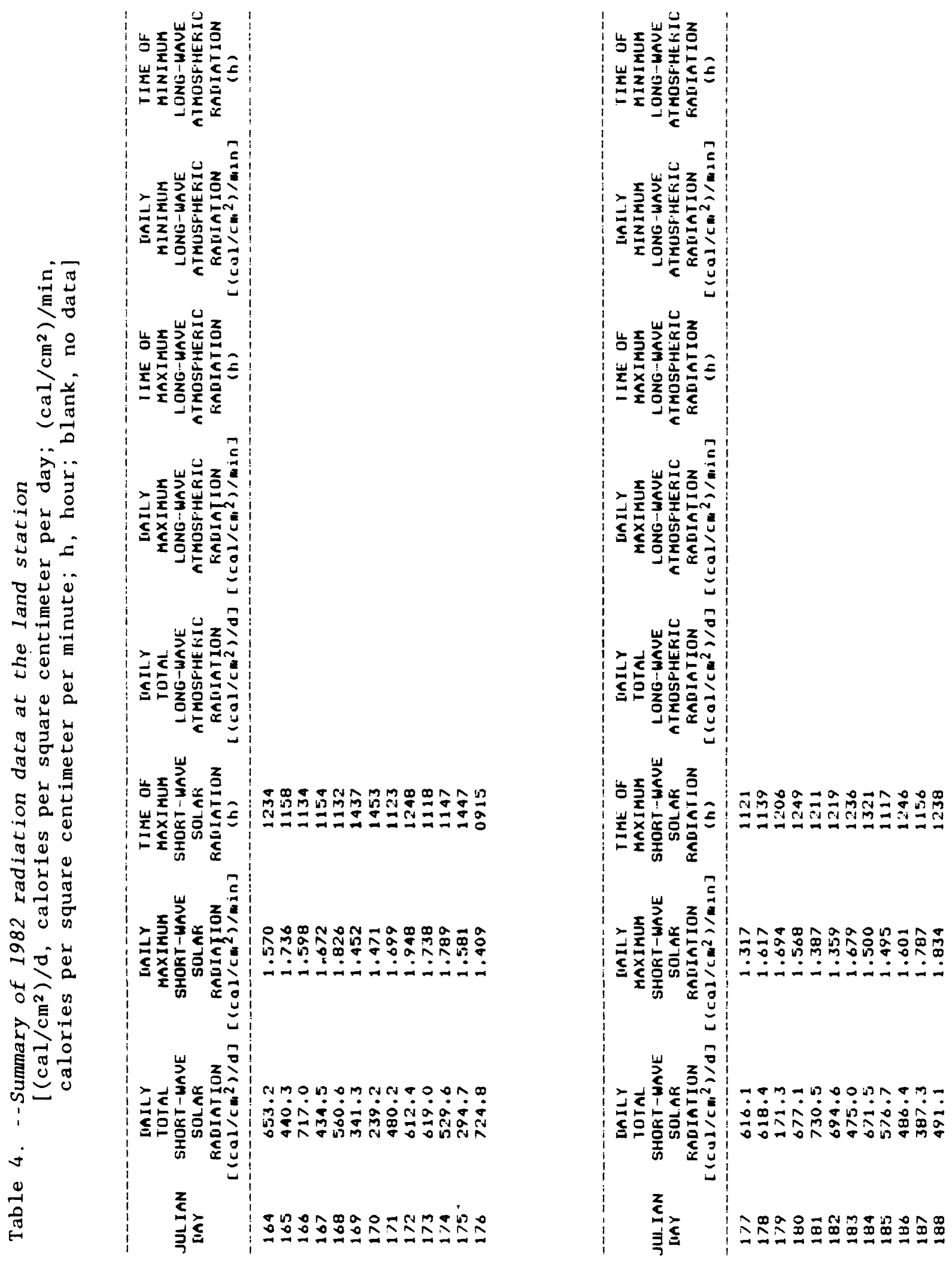

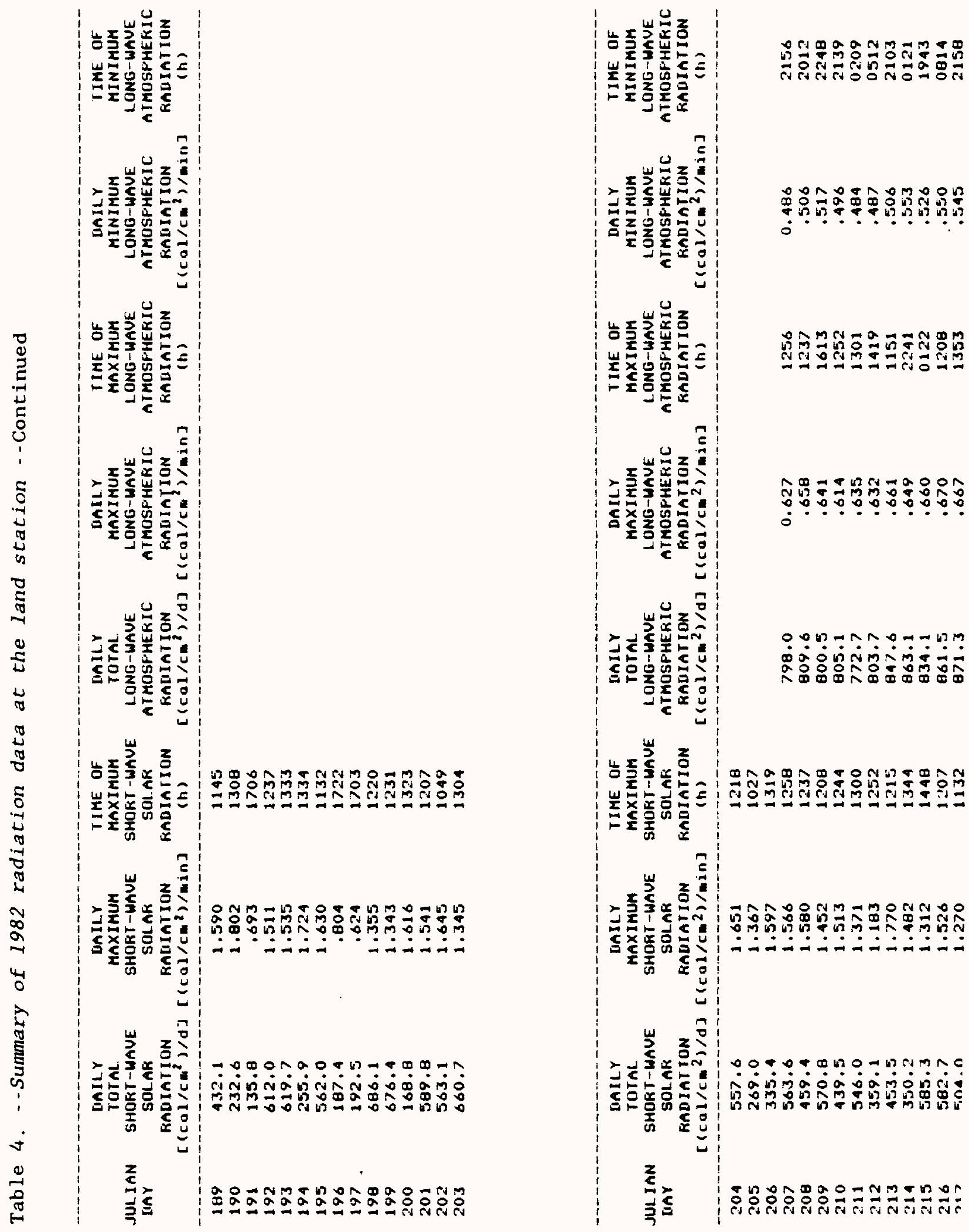

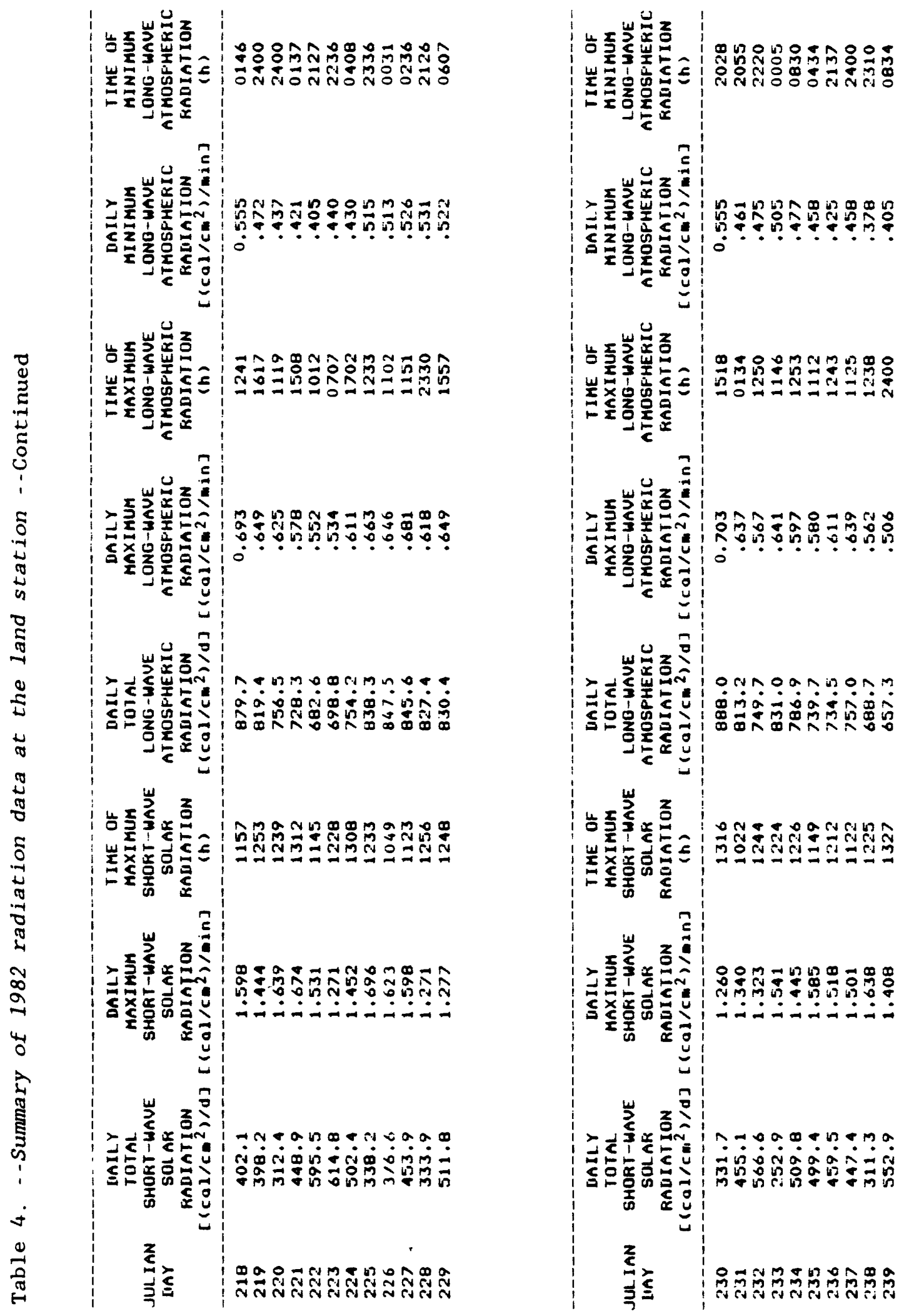

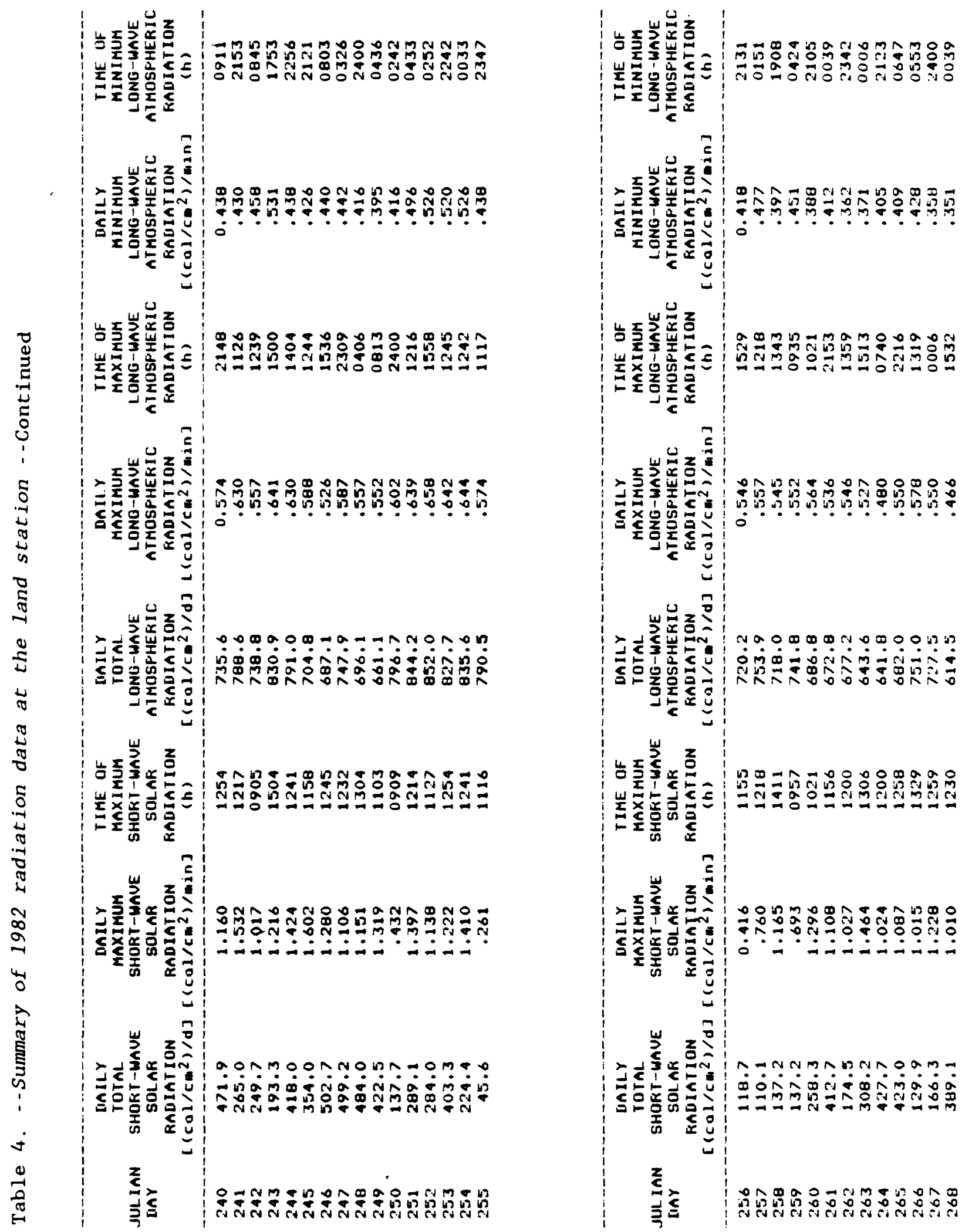

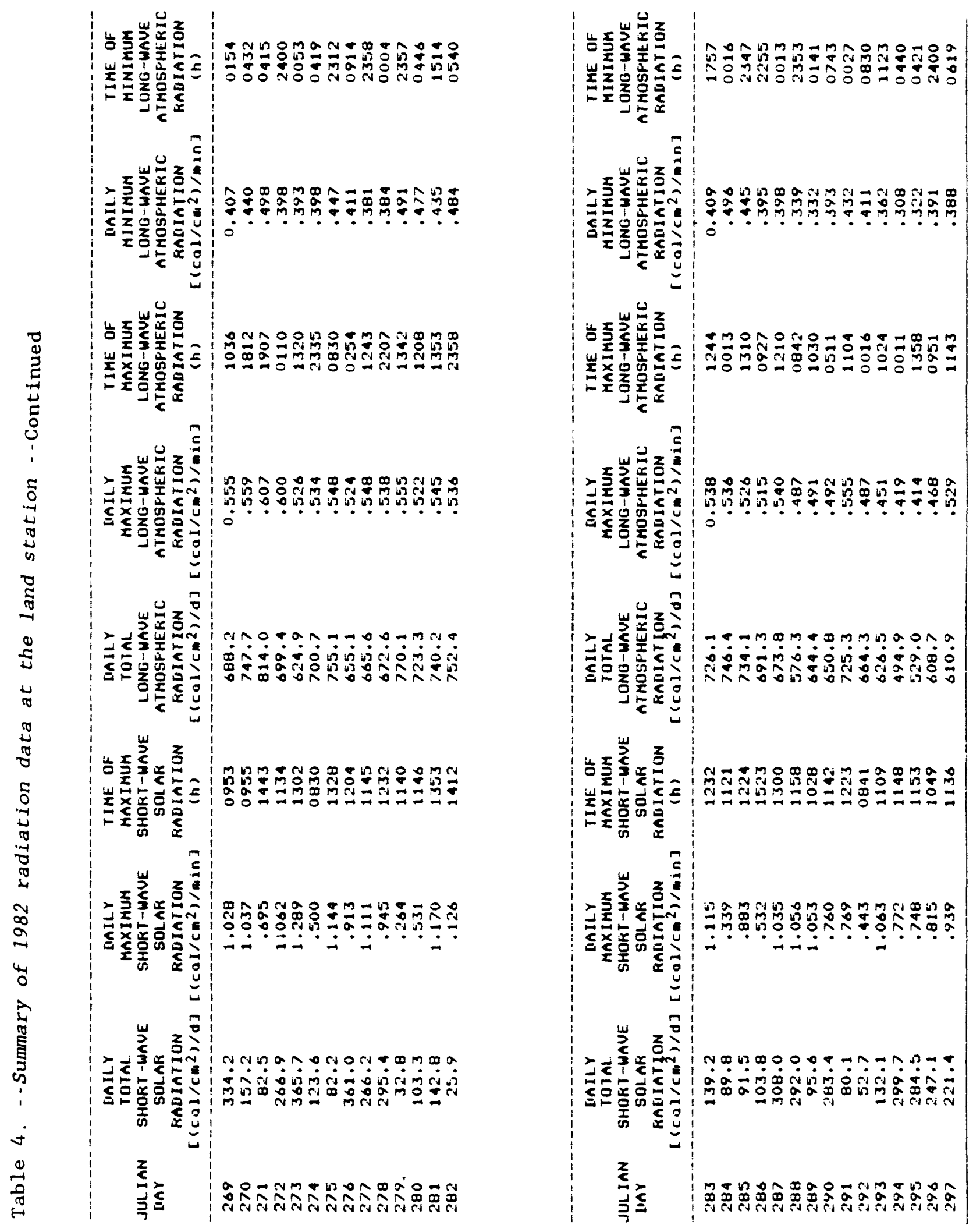

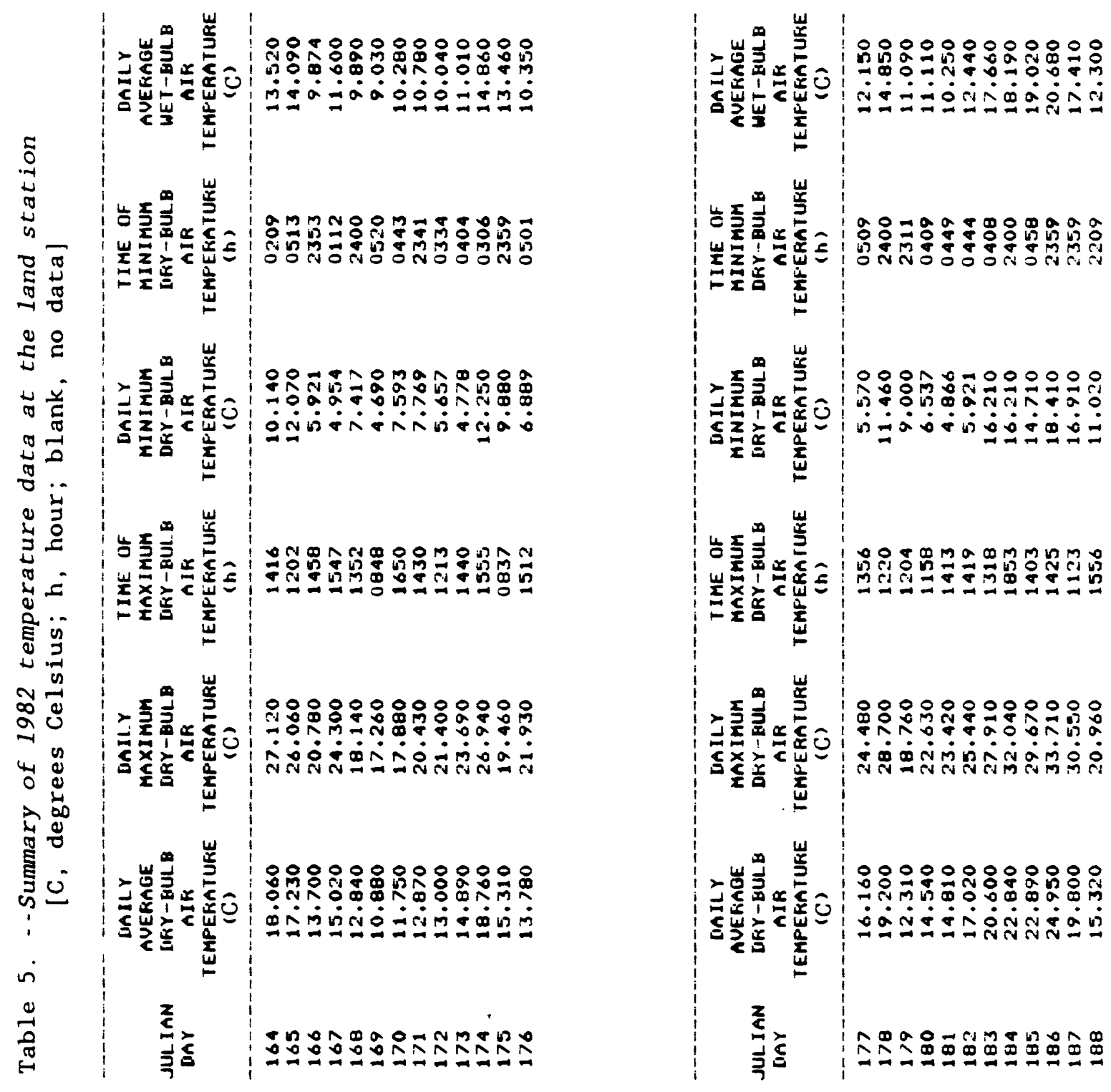

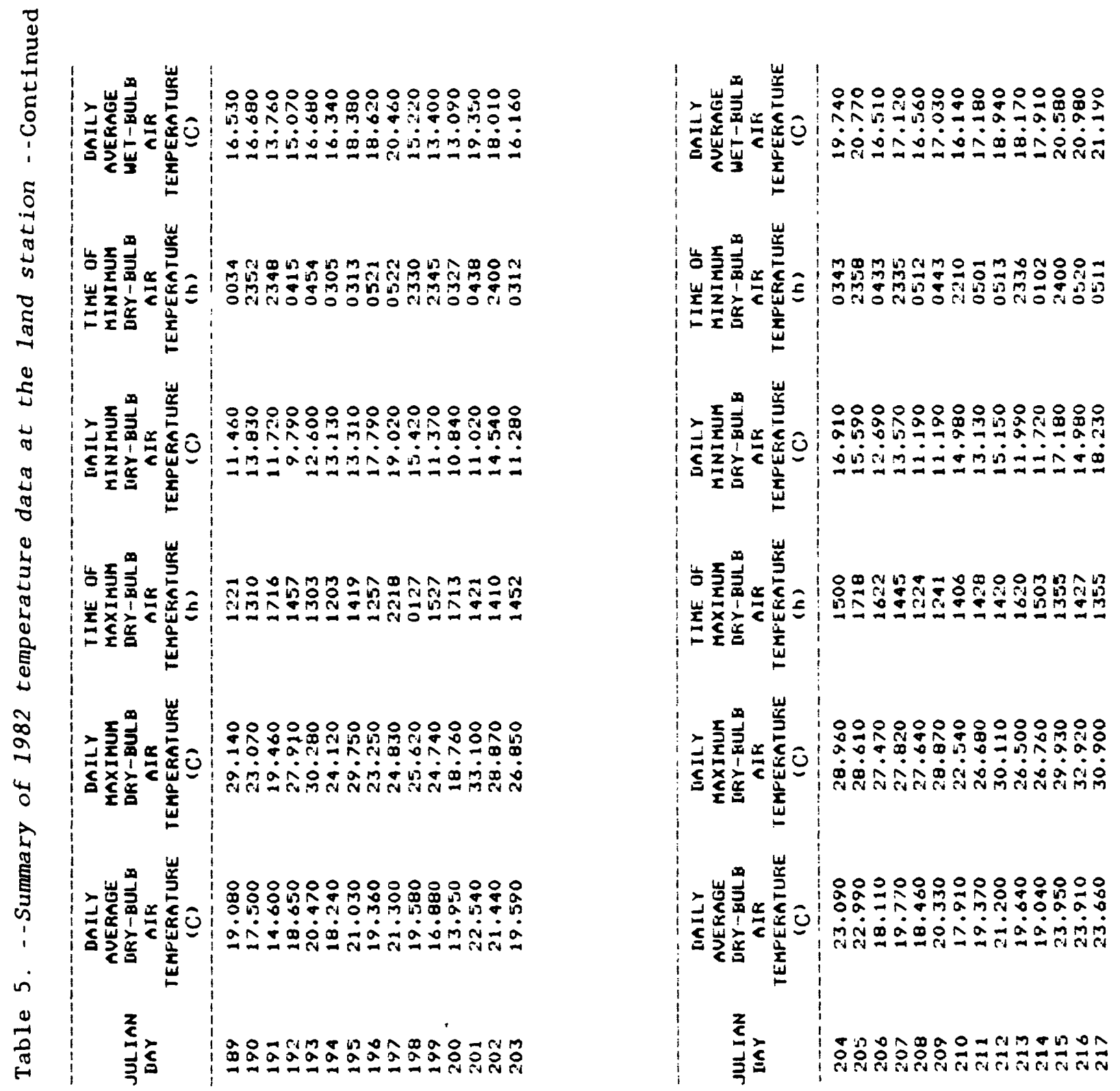

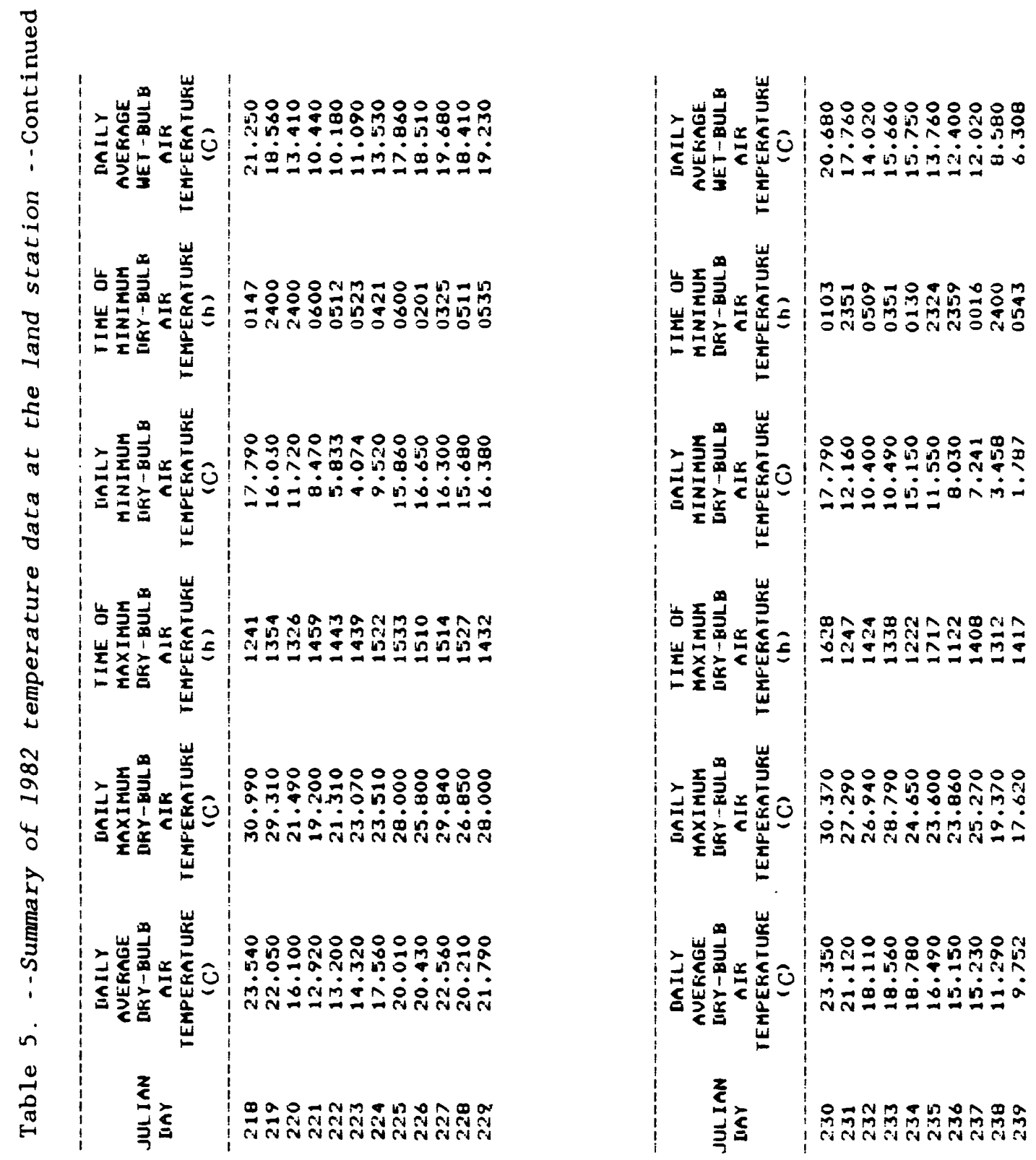

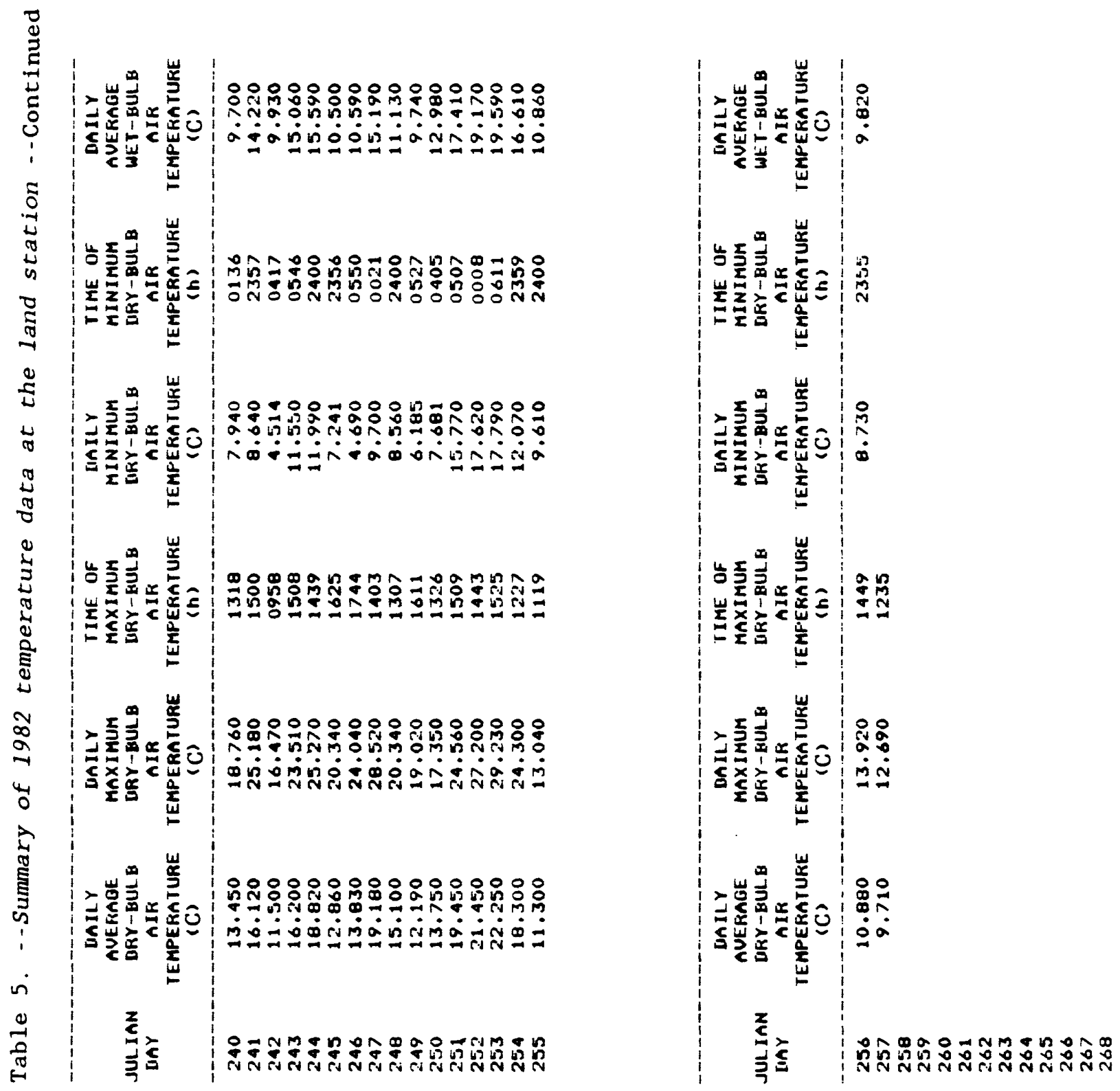

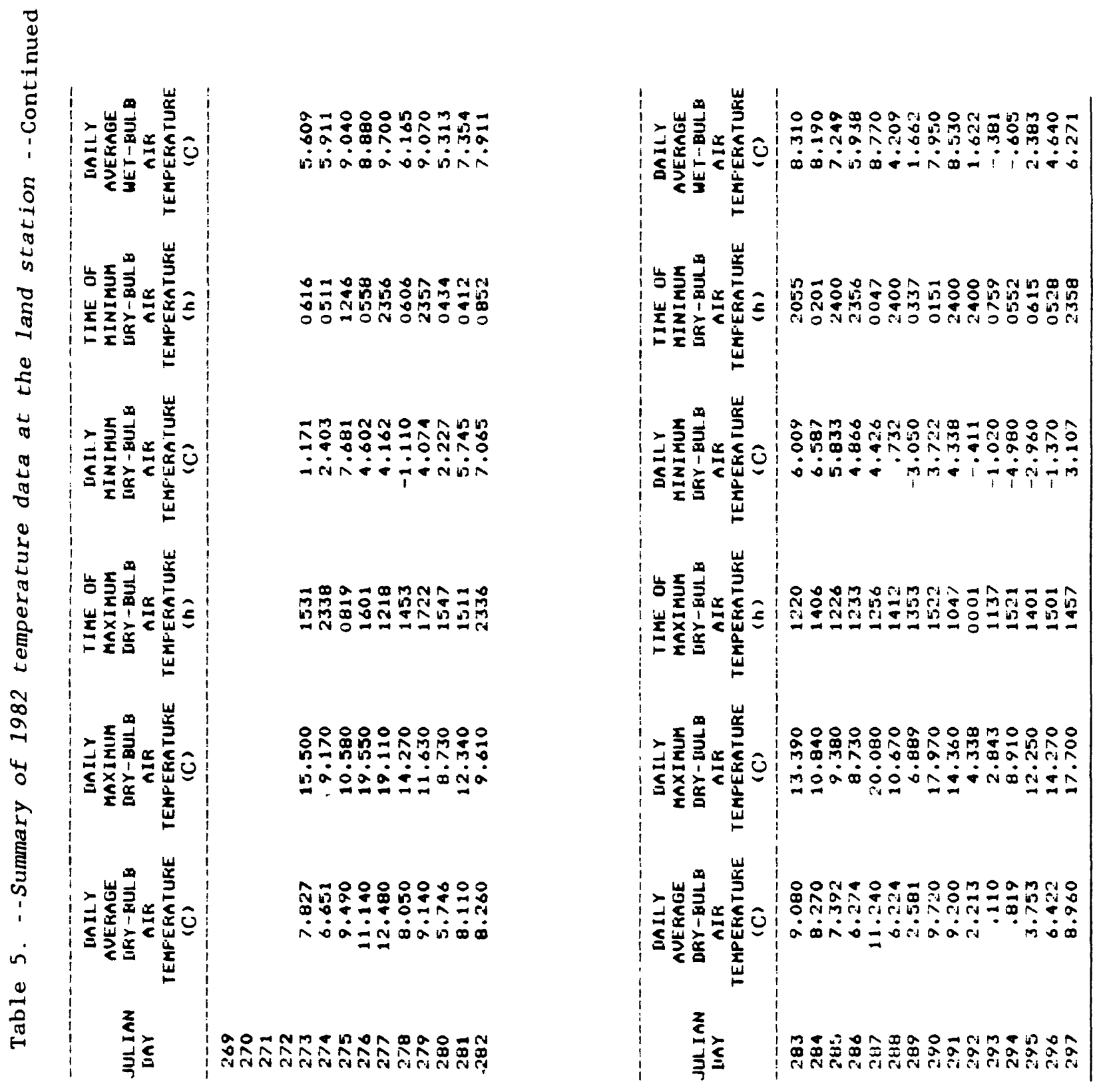


\begin{tabular}{|c|c|c|c|c|c|c|}
\hline $\begin{array}{l}\text { HE IGHT OF } \\
\text { ANEMOMF IER } \\
\text { AROUE LAKE } \\
\text { SURF ACE. } \\
(m)\end{array}$ & $\begin{array}{l}\text { JULL. I AN } \\
\text { IIAY }\end{array}$ & $\begin{array}{l}\text { IIAII.Y } \\
\text { AUERAGE } \\
\text { WINI } \\
\text { SFEEII } \\
\text { (mi/h) }\end{array}$ & $\begin{array}{l}\text { DAILY } \\
\text { MAXIMUM } \\
\text { WIND } \\
\text { SPEEII } \\
(m 1 / h)\end{array}$ & $\begin{array}{l}\text { TIME OF } \\
\text { MAXIMUM } \\
\text { WINI } \\
\text { SFEEII } \\
\text { (h) }\end{array}$ & $\begin{array}{l}\text { IIAILY } \\
\text { MINIMUM } \\
\text { WINII } \\
\text { SFEEI } \\
(\mathrm{mi} / \mathrm{h}) 1\end{array}$ & $\begin{array}{l}\text { TIME OF } \\
\text { MINIMUM } \\
\text { WINII } \\
\text { SFEEII } \\
(n)\end{array}$ \\
\hline 2 & $\begin{array}{l}164 \\
165 \\
166 \\
167 \\
168 \\
169 \\
170 \\
171 \\
172 \\
173 \\
174 \\
175 \\
176\end{array}$ & $\begin{array}{l}3.700 \\
1.942 \\
3.480 \\
2.175 \\
3.522 \\
1.520 \\
1.768 \\
3.682 \\
3.023 \\
1.962 \\
2.497 \\
3.927 \\
2.228\end{array}$ & $\begin{array}{r}15.200 \\
14.600 \\
14.720 \\
18.420 \\
15.420 \\
8.920 \\
9.790 \\
18.510 \\
13.390 \\
8.180 \\
12.930 \\
16.560 \\
10.220\end{array}$ & $\begin{array}{l}1204 \\
1402 \\
0945 \\
1042 \\
1058 \\
1616 \\
1512 \\
1537 \\
1323 \\
0949 \\
1615 \\
0150 \\
1530\end{array}$ & $\begin{array}{l}0.600 \\
.600 \\
.600 \\
.571 \\
.571 \\
.571 \\
.571 \\
.571 \\
.571 \\
.571 \\
.600 \\
.571 \\
.600\end{array}$ & $\begin{array}{l}2400 \\
2356 \\
2400 \\
2135 \\
2324 \\
1559 \\
2053 \\
2245 \\
0527 \\
2251 \\
2358 \\
2319 \\
2400\end{array}$ \\
\hline
\end{tabular}

\begin{tabular}{|c|c|c|c|c|c|c|}
\hline $\begin{array}{c}\text { HEIGHT OF } \\
\text { ANEMOME IE.K } \\
\text { AROUE L.AKE } \\
\text { SURFACE } \\
(m)\end{array}$ & $\begin{array}{l}\text { JUL I AN } \\
\text { IIAY }\end{array}$ & $\begin{array}{l}\text { UAILY } \\
\text { AUERAGE } \\
\text { WINI } \\
\text { SFEEI } \\
(m i / h)\end{array}$ & $\begin{array}{l}\text { LIAILY } \\
\text { MAXIMUM } \\
\text { WINII } \\
\text { SFEEII } \\
(\mathrm{mi} / \mathrm{h})\end{array}$ & $\begin{array}{l}\text { TIME OF } \\
\text { MAXIMUM } \\
\text { WINI } \\
\text { SFEEII } \\
\text { (h) }\end{array}$ & $\begin{array}{l}\text { DAILY } \\
\text { MINIMUM } \\
\text { WINI } \\
\text { SFEEI } \\
(\mathrm{mi} / \mathrm{h})\end{array}$ & $\begin{array}{l}\text { TIME OF } \\
\text { MINIMUM } \\
\text { WINII } \\
\text { SFEEII } \\
(h)\end{array}$ \\
\hline 2 & $\begin{array}{l}177 \\
1>8 \\
179 \\
180 \\
181 \\
182 \\
183 \\
184 \\
185 \\
186 \\
187 \\
188\end{array}$ & $\begin{array}{l}1.802 \\
2.374 \\
3.365 \\
2.635 \\
1.972 \\
2.379 \\
2.207 \\
3.606 \\
3.334 \\
4.423 \\
1.736 \\
4.500\end{array}$ & $\begin{array}{r}8.460 \\
11.350 \\
14.120 \\
12.110 \\
10.590 \\
9.030 \\
10.360 \\
16.470 \\
17.430 \\
25.070 \\
14.600 \\
18.850\end{array}$ & $\begin{array}{l}1643 \\
1456 \\
1505 \\
1222 \\
1556 \\
1319 \\
1420 \\
1326 \\
2259 \\
2352 \\
0004 \\
1009\end{array}$ & $\begin{array}{r}0.600 \\
.571 \\
.600 \\
.571 \\
.571 \\
.600 \\
.600 \\
.600 \\
.571 \\
.600 \\
.600 \\
.600\end{array}$ & $\begin{array}{l}2352 \\
0337 \\
2359 \\
2111 \\
2048 \\
2107 \\
2351 \\
2400 \\
0550 \\
2259 \\
2342 \\
2359\end{array}$ \\
\hline
\end{tabular}


Table 6. --Sumary of 1982 wind-speed data at the land station --Continued

\begin{tabular}{|c|c|c|c|c|c|c|}
\hline $\begin{array}{c}\text { HEIGHT OF } \\
\text { ANEMOMETER } \\
\text { ABOUE LAKE } \\
\text { SURF ACE } \\
(m)\end{array}$ & $\underset{\text { JIAY }}{\text { JULN }}$ & $\begin{array}{l}\text { IIAILY } \\
\text { AUERAGE } \\
\text { WINII } \\
\text { SPEEI } \\
\text { (ni/h) }\end{array}$ & $\begin{array}{l}\text { DAILY } \\
\text { MAXIMUM } \\
\text { WINI } \\
\text { SFEED } \\
(m i / h)\end{array}$ & $\begin{array}{l}\text { TIME OF } \\
\text { MAXIMUM } \\
\text { WIND } \\
\text { SPEED } \\
\text { (h) }\end{array}$ & $\begin{array}{l}\text { DAILY } \\
\text { MINIMUM } \\
\text { WIND } \\
\text { SPEEI } \\
(m i / h)\end{array}$ & $\begin{array}{l}\text { TIME OF } \\
\text { MINIMUM } \\
\text { WIND } \\
\text { SFEER } \\
\text { (h) }\end{array}$ \\
\hline 2 & $\begin{array}{l}189 \\
190 \\
191 \\
192 \\
193 \\
194 \\
195 \\
196 \\
197 \\
198 \\
199 \\
200 \\
201 \\
202 \\
203\end{array}$ & $\begin{array}{l}2.112 \\
3.053 \\
2.514 \\
2.636 \\
2.229 \\
1.549 \\
2.271 \\
3.079 \\
3.065 \\
5.561 \\
2.852 \\
2.584 \\
1.901 \\
2.461 \\
2.480\end{array}$ & $\begin{array}{r}10.440 \\
10.560 \\
9.710 \\
15.230 \\
12.590 \\
7.476 \\
9.110 \\
11.830 \\
11.210 \\
20.720 \\
12.140 \\
10.640 \\
17.830 \\
10.920 \\
9.030\end{array}$ & $\begin{array}{l}1124 \\
1820 \\
0737 \\
1231 \\
1613 \\
1350 \\
2227 \\
1410 \\
1731 \\
0202 \\
1229 \\
0650 \\
2238 \\
1655 \\
0824\end{array}$ & $\begin{array}{l}0.571 \\
.600 \\
.600 \\
.571 \\
.571 \\
.571 \\
.600 \\
.600 \\
.600 \\
.600 \\
.571 \\
.571 \\
.600 \\
.600 \\
.600\end{array}$ & $\begin{array}{l}0023 \\
1126 \\
2400 \\
2221 \\
2218 \\
1023 \\
2316 \\
2224 \\
2255 \\
2353 \\
0113 \\
0153 \\
2400 \\
2344 \\
2400\end{array}$ \\
\hline
\end{tabular}

\begin{tabular}{|c|c|c|c|c|c|c|}
\hline $\begin{array}{c}\text { HEIGHT OF } \\
\text { ANEMOMETER } \\
\text { ABOUE LAKE } \\
\text { SURFACE } \\
(m)\end{array}$ & $\begin{array}{l}\text { JUL IAN } \\
\text { IIAY }\end{array}$ & $\begin{array}{l}\text { DAILY } \\
\text { AUERAGE } \\
\text { WINE } \\
\text { SPEED } \\
\text { (ni/h) }\end{array}$ & $\begin{array}{l}\text { IIAILY } \\
\text { MAXIMUM } \\
\text { WIND } \\
\text { SFEEI } \\
(m \perp / h)\end{array}$ & $\begin{array}{l}\text { TIME OF } \\
\text { MAXIMUM } \\
\text { WIND } \\
\text { SPEEI } \\
\text { (h) }\end{array}$ & $\begin{array}{l}\text { DAILY } \\
\text { MINIMUM } \\
\text { WINI } \\
\text { SFEED } \\
\text { (mi/h) }\end{array}$ & $\begin{array}{c}\text { TIME OF } \\
\text { MINIMUM } \\
\text { WIND } \\
\text { SFEED } \\
(h)\end{array}$ \\
\hline 2 & $\begin{array}{l}204 \\
205 \\
206 \\
207 \\
208 \\
209 \\
210 \\
211 \\
212 \\
213 \\
214 \\
215 \\
216 \\
217\end{array}$ & $\begin{array}{l}2.885 \\
2.768 \\
1.629 \\
2.028 \\
1.677 \\
1.420 \\
4.067 \\
2.664 \\
1.853 \\
3.230 \\
4.247 \\
2.631 \\
2.042 \\
1.959\end{array}$ & $\begin{array}{r}10.610 \\
15.340 \\
10.870 \\
9.990 \\
7.618 \\
13.700 \\
18.680 \\
13.840 \\
10.750 \\
12.450 \\
14.460 \\
25.390 \\
7.193 \\
7.788\end{array}$ & $\begin{array}{l}1309 \\
0459 \\
1407 \\
1002 \\
1321 \\
2020 \\
0926 \\
1109 \\
1225 \\
2019 \\
1044 \\
0125 \\
2154 \\
1329\end{array}$ & $\begin{array}{l}0.600 \\
.600 \\
.600 \\
.571 \\
.571 \\
.571 \\
.600 \\
.571 \\
.486 \\
.600 \\
.600 \\
.600 \\
.571 \\
.571\end{array}$ & $\begin{array}{l}2329 \\
2400 \\
2400 \\
0653 \\
0709 \\
2239 \\
2308 \\
1734 \\
0559 \\
0713 \\
2303 \\
2355 \\
1321 \\
2156\end{array}$ \\
\hline
\end{tabular}


Table 6. --Summary of 1982 wind-speed data at the land station --Continued

\begin{tabular}{|c|c|c|c|c|c|c|}
\hline $\begin{array}{l}\text { HE IGHT OF } \\
\text { ANEMOME TER } \\
\text { AEOUE LAKE } \\
\text { SURFACE } \\
(m)\end{array}$ & $\begin{array}{l}\text { JUL IAN } \\
\text { IIAY }\end{array}$ & $\begin{array}{l}\text { DAILY } \\
\text { AUERAGE } \\
\text { WINI } \\
\text { SFEEI } \\
\left(m_{1} / h\right)\end{array}$ & $\begin{array}{l}\text { IIAILY } \\
\text { MAXIMUM } \\
\text { WINI } \\
\text { SFEEI } \\
\left(M_{1} 1 / h\right)\end{array}$ & $\begin{array}{l}\text { TIME OF } \\
\text { MAXIMUM } \\
\text { WINR } \\
\text { SFEED } \\
\text { (h) }\end{array}$ & $\begin{array}{l}\text { DIAILY } \\
\text { MINIMUM } \\
\text { WIND } \\
\text { SPEED } \\
\left(m_{1} / h\right)\end{array}$ & $\begin{array}{l}\text { TIME OF } \\
\text { MINIMUM } \\
\text { WIND } \\
\text { SPEE[I } \\
(h)\end{array}$ \\
\hline 2 & $\begin{array}{l}218 \\
219 \\
220 \\
221 \\
222 \\
223 \\
224 \\
225 \\
226 \\
227 \\
228 \\
229\end{array}$ & $\begin{array}{l}1.789 \\
3.447 \\
5.895 \\
4.135 \\
2.225 \\
2.116 \\
3.057 \\
1.788 \\
2.087 \\
1.867 \\
1.727 \\
1.992\end{array}$ & $\begin{array}{r}10.750 \\
14.940 \\
19.130 \\
15.370 \\
9.230 \\
8.860 \\
11.490 \\
8.380 \\
7.675 \\
10.920 \\
9.930 \\
9.040\end{array}$ & $\begin{array}{l}0913 \\
1556 \\
0928 \\
1219 \\
1551 \\
1414 \\
1200 \\
1212 \\
1240 \\
0915 \\
1033 \\
1300\end{array}$ & $\begin{array}{l}0.571 \\
.600 \\
.600 \\
.571 \\
.571 \\
.571 \\
.600 \\
.600 \\
.571 \\
.571 \\
.571 \\
.600\end{array}$ & $\begin{array}{l}2103 \\
2031 \\
1852 \\
2136 \\
0154 \\
0642 \\
2330 \\
2400 \\
0915 \\
1925 \\
0157 \\
2357\end{array}$ \\
\hline
\end{tabular}

\begin{tabular}{|c|c|c|c|c|c|c|}
\hline $\begin{array}{l}\text { HEIGHT OF } \\
\text { ANEMOME.TER } \\
\text { ABOUE LARE } \\
\text { SURFACE } \\
(m)\end{array}$ & $\begin{array}{l}\text { JUL JAAN } \\
\text { IIAY }\end{array}$ & $\begin{array}{l}\text { IIAILY } \\
\text { AUERAGE } \\
\text { WINII } \\
\text { SFEEI } \\
(m 1 / h)\end{array}$ & $\begin{array}{l}\text { IAAILY } \\
\text { MAXIMUM } \\
\text { WINII } \\
\text { SFEEII } \\
(m i / h)\end{array}$ & $\begin{array}{l}\text { TIME OF } \\
\text { MAXIMUM } \\
\text { WINI } \\
\text { SFEEI } \\
(h)\end{array}$ & $\begin{array}{l}\text { IIAILY } \\
\text { MINIMUM } \\
\text { WINI } \\
\text { SFEED } \\
(\mathrm{mi} / \mathrm{h})\end{array}$ & $\begin{array}{c}\text { TIME DF } \\
\text { MINIMUM } \\
\text { WIND } \\
\text { SFEED } \\
(h)\end{array}$ \\
\hline 2 & $\begin{array}{l}230 \\
231 \\
232 \\
233 \\
234 \\
235 \\
240 \\
237 \\
238 \\
239\end{array}$ & $\begin{array}{l}2.488 \\
2.457 \\
2.690 \\
2.099 \\
2.841 \\
2.347 \\
.4 .88 \\
3.411 \\
2.415 \\
2.385\end{array}$ & $\begin{array}{l}22.220 \\
13.080 \\
13.840 \\
18.650 \\
11.010 \\
10.730 \\
19.730 \\
19.500 \\
11.380 \\
12.370\end{array}$ & $\begin{array}{l}1927 \\
1255 \\
1322 \\
1933 \\
1300 \\
1126 \\
1259 \\
1111 \\
1708 \\
0946\end{array}$ & $\begin{array}{l}0.600 \\
.571 \\
.571 \\
.571 \\
.600 \\
.600 \\
.571 \\
.600 \\
.600 \\
.571\end{array}$ & $\begin{array}{l}2353 \\
0452 \\
0135 \\
0733 \\
2359 \\
2359 \\
0137 \\
2326 \\
2400 \\
0319\end{array}$ \\
\hline
\end{tabular}


Table 6. --Summary of 1982 wind-speed data at the land station --Continued

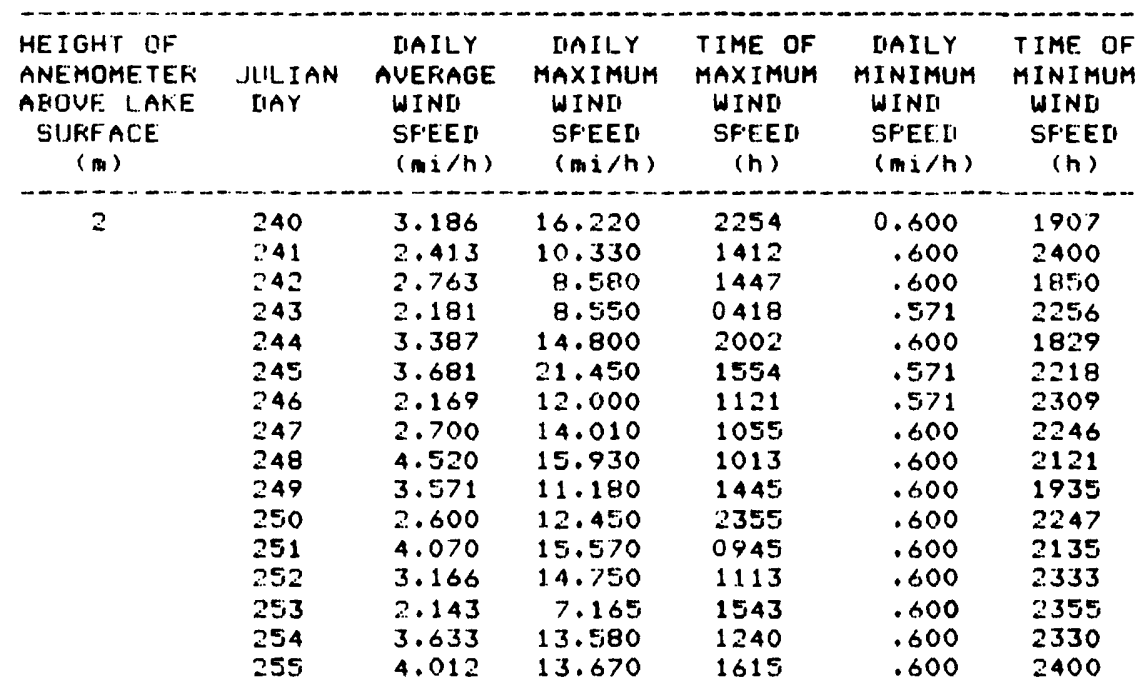

\begin{tabular}{|c|c|c|c|c|c|c|}
\hline $\begin{array}{l}\text { HE IGHT DF } \\
\text { ANEMOMETER } \\
\text { AEOUE LAKE } \\
\text { SIII: AI \& } \\
\text { (m) }\end{array}$ & $\begin{array}{l}\text { JUL IAN } \\
\text { IIAY }\end{array}$ & $\begin{array}{l}\text { IUAILY } \\
\text { AUERAGE } \\
\text { WIND } \\
\text { SPEEII } \\
(\mathrm{mi} / \mathrm{h})\end{array}$ & $\begin{array}{l}\text { IIAILY } \\
\text { MAXIMUM } \\
\text { WINU } \\
\text { SPEED } \\
(\mathrm{mi} / \mathrm{h})\end{array}$ & $\begin{array}{l}\text { TIME OF } \\
\text { MAXIMUM } \\
\text { WIND } \\
\text { SFEELI } \\
\text { (h) }\end{array}$ & $\begin{array}{l}\text { IIAILY } \\
\text { MINIMUM } \\
\text { WINU } \\
\text { SFEEII } \\
\left(m_{1} / h\right)\end{array}$ & $\begin{array}{l}\text { TIME OF } \\
\text { MINIMUM } \\
\text { WINU } \\
\text { SF'EEII } \\
\text { (h) }\end{array}$ \\
\hline$?$ & $\begin{array}{l}256 \\
257 \\
258 \\
259 \\
260 \\
261 \\
262 \\
263 \\
264 \\
265 \\
266 \\
267 \\
268\end{array}$ & $\begin{array}{l}3.111 \\
4.309 \\
2.039 \\
1.888 \\
3.200 \\
2.816 \\
4.390 \\
2.393 \\
1.504 \\
3.023 \\
4.039 \\
4.493 \\
2.229\end{array}$ & $\begin{array}{r}11.410 \\
15.480 \\
7.392 \\
10.870 \\
12.510 \\
15.400 \\
14.860 \\
13.810 \\
6.514 \\
14.430 \\
13.670 \\
16.870 \\
12.030\end{array}$ & $\begin{array}{l}1426 \\
1443 \\
1354 \\
2233 \\
1316 \\
1257 \\
1328 \\
1058 \\
0949 \\
1703 \\
1004 \\
1404 \\
1544\end{array}$ & $\begin{array}{l}0.600 \\
.600 \\
.571 \\
.571 \\
.600 \\
.571 \\
.571 \\
.571 \\
.571 \\
.600 \\
.571 \\
.600 \\
.571\end{array}$ & $\begin{array}{l}2345 \\
2121 \\
2250 \\
1815 \\
2400 \\
2345 \\
0335 \\
2249 \\
2131 \\
2152 \\
0138 \\
2400 \\
0741\end{array}$ \\
\hline
\end{tabular}


Table 6. --Sumnary of 1982 wind-speed data at the land station --Continued

\begin{tabular}{|c|c|c|c|c|c|c|}
\hline $\begin{array}{l}\text { HEIGHT OF } \\
\text { ANEMOMETER } \\
\text { ABQUE LAKE } \\
\text { SUFFACE } \\
(\mathrm{m})\end{array}$ & $\begin{array}{l}\text { JUL I AN } \\
\text { IIAY }\end{array}$ & $\begin{array}{l}\text { IIAILY } \\
\text { AUEFIAGE } \\
\text { WINII } \\
\text { SFEEI } \\
\left(n_{1} 1 / n\right)\end{array}$ & $\begin{array}{l}\text { IIAILY } \\
\text { MAXIMLIM } \\
\text { WINII } \\
\text { SFEEII } \\
(m i / h)\end{array}$ & $\begin{array}{l}\text { TIME OF } \\
\text { MAXIMUM } \\
\text { WINII } \\
\text { SFEE[I } \\
\text { (h) }\end{array}$ & $\begin{array}{l}\text { [IAILY } \\
\text { MINIMUM } \\
\text { WINU } \\
\text { SPEEI } \\
\left(m_{1} / h\right)\end{array}$ & $\begin{array}{l}\text { TIME OF } \\
\text { MINIMUM } \\
\text { WINII } \\
\text { SFEEL } \\
\text { (h) }\end{array}$ \\
\hline- & -- & ----- & $\cdots-$ & --- & ----- & $-\cdots--$ \\
\hline \multirow[t]{8}{*}{2} & $\begin{array}{l}269 \\
270 \\
271\end{array}$ & $\begin{array}{l}5.534 \\
5.387\end{array}$ & $\begin{array}{l}13.130 \\
17.290\end{array}$ & $\begin{array}{l}1229 \\
2118\end{array}$ & $\begin{array}{r}0.571 \\
.571\end{array}$ & $\begin{array}{l}2044 \\
0427\end{array}$ \\
\hline & $\begin{array}{l}272 \\
273\end{array}$ & $\begin{array}{l}2.355 \\
3.038\end{array}$ & $\begin{array}{l}12.930 \\
12.590\end{array}$ & $\begin{array}{l}0001 \\
2119\end{array}$ & $\begin{array}{r}.571 \\
.571\end{array}$ & $\begin{array}{l}0 \geq 34 \\
0730\end{array}$ \\
\hline & 274 & 4.848 & 14.150 & 1959 & .571 & 0513 \\
\hline & 275 & 3.839 & 14.490 & 1024 & .571 & 1938 \\
\hline & 277 & 4.286 & 15.170 & 1116 & .600 & 2400 \\
\hline & 278 & 4.184 & 16.670 & 2057 & .600 & 1904 \\
\hline & 279 & 5.394 & 18.570 & 1335 & .600 & 2334 \\
\hline & $\begin{array}{l}280 \\
281 \\
282\end{array}$ & 3.687 & 13.900 & 1046 & .543 & 1926 \\
\hline
\end{tabular}

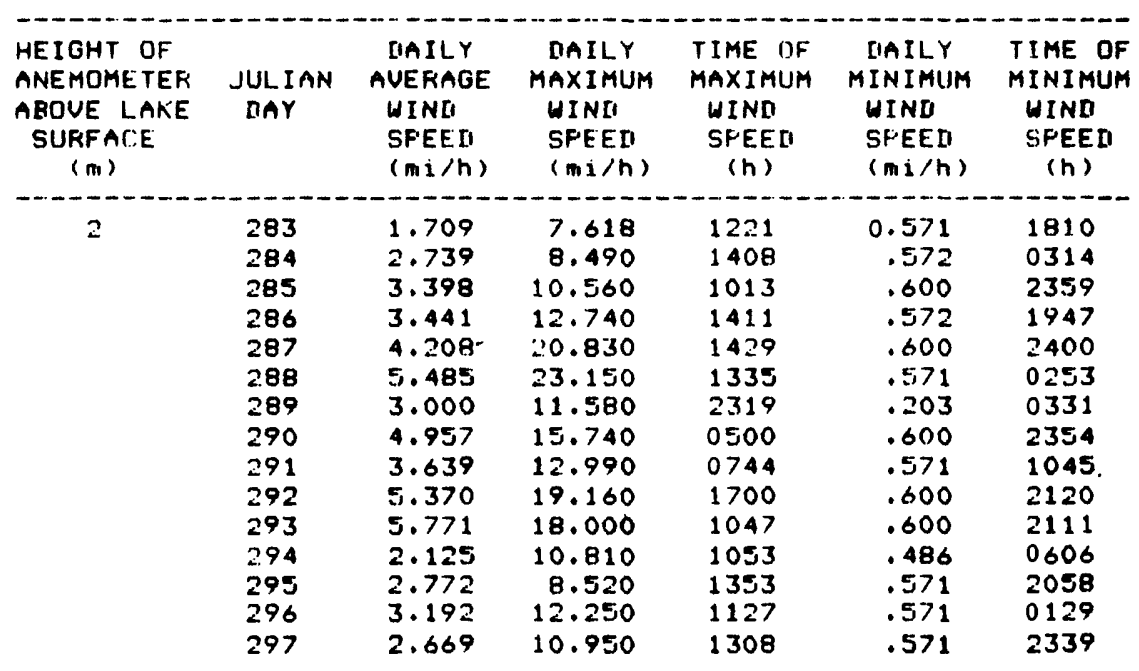

1 Values of 0.600 or 0.571 are threshold values that represent the minimum windspeed required to turn the anemometer cups. 